\title{
NONLINEAR DEGENERATE INTEGRO-PARTIAL DIFFERENTIAL EVOLUTION EQUATIONS RELATED TO GEOMETRIC LÉVY PROCESSES AND APPLICATIONS TO BACKWARD STOCHASTIC DIFFERENTIAL EQUATIONS
}

\author{
ANNA LISA AMADORI, KENNETH H. KARLSEN, AND CLAUDIA LA CHIOMA
}

\begin{abstract}
We prove a comparison principle for unbounded semicontinuous viscosity sub- and supersolutions of nonlinear degenerate parabolic integro-partial differential equations coming from applications in mathematical finance in which geometric Lévy processes act as the underlying stochastic processes for the assets dynamics. As a consequence of the "geometric form" of these processes, the comparison principle holds without assigning spatial boundary data. We present applications of our result to (i) backward stochastic differential equations and (ii) pricing of European and American derivatives via backward stochastic differential equations. Regarding (i), we extend previous results on backward stochastic differential equations in a Lévy setting and the connection to semilinear integro-partial differential equations.
\end{abstract}

\section{INTRODUCTION}

In recent years there has been an interest in developing viscosity solution theory [11, 12] for integro-partial differential equations. The relevance of integro-partial differential equations can be motivated by their many applications in mathematical finance in which Lévy processes act as the underlying stochastic processes. Empirical work shows that the normal distribution poorly fits the logreturn data for, e.g., stock prices. Among other things the data show heavier tails than predicted by the normal distribution, and it has in recent years been suggested to model logreturns by generalized hyperbolic distributions (see the references in $[6,7,8,10,26,9]$ for relevant works).

In this paper we are interested in viscosity solutions of nonlinear degenerate parabolic integro-partial differential equations with a given terminal condition $u_{T}$ :

$$
\begin{array}{cc}
-\partial_{t} u+H\left(x, t, u, \mathbf{x} D u, \mathbf{x} D^{2} u \mathbf{x}^{T}, \mathcal{J} u\right)=0, & (x, t) \in \Pi_{T}, \\
u(x, T)=u_{T}(x), & x \in \Pi,
\end{array}
$$

where $\Pi=(0, \infty)^{\mathrm{N}}, \Pi_{T}=\Pi \times[0, T), \mathbf{x}=\operatorname{diag}\left(x_{1}, \cdots, x_{\mathrm{N}}\right)$, and $\mathcal{J} u$ is an integro-differential operator given by

$$
\mathcal{J} u(x, t)=\int_{E}[u(x+\mathbf{x} \beta(x, t, z), t)-u(x, t)-\mathbf{x} \beta(x, t, z) \cdot D u(x, t)] \nu(d z) .
$$

In (1.3), $\nu$ is a given Radon measure on $E=\mathbb{R}^{2} \backslash\{0\}$ (the so-called Lévy measure), which may possess a second order singularity at the origin, while $\beta$ is a given function of $(x, t, z) \in \Pi_{T} \times E$ with values in $(-1, \infty)^{\mathrm{N}}$. Without introducing any new substantial difficulty, it is possible to deal also with some different integral operator of the type

$$
\mathcal{I} u(x, t)=\int_{E}[u(x+\mathbf{x} \beta(x, t, z), t)-u(x, t)] \gamma(x, t, z) \nu(d z),
$$

where $\gamma$ is a given function of $(x, t, z) \in \Pi_{T} \times E$ with values in $(0, \infty)$. Precise assumptions on the coefficients in (1.1), (1.3), and (1.4) are postponed to Section 2.

Because (1.1) may be degenerate and $\nu$ is allowed to have a second order singularity at the origin $\left(\mathcal{I}\right.$ and $\mathcal{J}$ are typically well defined on suitable subsets of the spaces of $\mathcal{C}^{1}$ and

Date: May 21, 2004

1991 Mathematics Subject Classification. Primary: 45K55; Secondary: 49L20, 60H10.

Key words and phrases. integro-partial differential equations, viscosity solutions, comparison principles, Lévy processes, backward stochastic differential equations, option pricing. 
$\mathcal{C}^{2}$ functions, respectively), it seems natural to study (1.1) in the framework of viscosity solutions. We refer to $[14,15]$ for an investigation of integro-partial differential equations in the framework of Green functions and more regular solutions in Sobolev spaces.

Various existence and comparison/uniqueness results for viscosity solutions of integropartial differential equations of first order (i.e., no local second order term) can be found in $[30,31,27,28,6]$. When the Lévy measure is bounded, general existence and comparison/uniqueness results for semicontinuous unbounded viscosity solutions of second order degenerate parabolic integro-partial differential equations are given in $[1,2,3]$. When the Lévy measure is unbounded near the origin, the existence and uniqueness of unbounded viscosity solutions of (systems of) semilinear degenerate parabolic integro-partial differential equations in $\mathbb{R}^{N}$ is proved in [4]. An existence result and a comparison principle among uniformly continuous and at most linearly growing viscosity sub- and supersolutions of fully nonlinear parabolic integro-partial differential equations of the Bellman type are proved in [25], see also [21] for some other existence results. The Bellman equations (variational inequalities) associated with some singular stochastic control problems arising in finance are studied in $[7,8]$. In [16], the authors prove a "non-local" maximum principle for semicontinuous viscosity sub- and supersolutions of integro-partial differential equations, which should be compared with the "local" maximum principle for semicontinuous functions [11]. Such a result can be used to obtain various comparison results for integro-partial differential equations, and is also used herein.

The contributions of the present paper can be summarized as follows:

1. We prove in Section 2 that one can have a comparison principle for unbounded semicontinuous viscosity sub- and supersolutions of (1.1)-(1.2) without assigning boundary data on $\partial \Pi$. The special structure of our problem that allows for this is that

$$
\mathbf{x} D u=\left(x_{1} u_{x_{1}}, \ldots, x_{\mathrm{N}} u_{x_{\mathrm{N}}}\right), \quad \mathbf{x} D^{2} u \mathbf{x}^{T}=\left(\begin{array}{ccc}
x_{1}^{2} u_{x_{1} x_{1}} & \cdots & x_{1} x_{\mathrm{N}} u_{x_{1} x_{\mathrm{N}}} u \\
\vdots & & \vdots \\
x_{1} x_{\mathrm{N}} u_{x_{1} x_{\mathrm{N}}} & \cdots & x_{\mathrm{N}}^{2} u_{x_{\mathrm{N}} x_{\mathrm{N}}}
\end{array}\right)
$$

occur respectively in the gradient and matrix slots of (1.1), while

$$
\mathbf{x} \beta=\left(x_{1} \beta_{1}, \ldots, x_{\mathrm{N}} \beta_{\mathrm{N}}\right)
$$

occurs in the integral operators (1.3) and (1.4). This structure arises naturally in finance applications with geometric Brownian motions, or more generally geometric Lévy processes, as the underlying stochastic processes for the assets dynamics (we will come back to this below). In the case of no integral operator, the problem (1.1)-(1.2) is equivalent to a Cauchy problem, up to a logarithmic change of variables. It is widely known that imposing some blow-up rate both at $\partial \Pi$ and at infinity yields uniqueness. This is in general not our case, because changing variables modifies the structure of the integral operator (1.3). So (1.1) presents two different kinds of degeneracies: one at the boundary $\partial \Pi$ (where $\mathbf{x} D^{2} u \mathbf{x}$ vanishes), and one at the interior points (where $H$ is only assumed to be degenerate elliptic). Boundary value problems for non-uniformly parabolic equations have been studied by many authors along the lines of [22]. In this framework, we mention [33] for some different integropartial differential equation. In the viscosity solution setting, comparison principles allowing degeneracy can be found in [5] in the context of Bellman equations (without an integro operator). The new aspects herein are that (i) we prove such a result for a large class of integro-partial differential equations, (ii) we deal with an unbounded set with nonsmooth boundary, (iii) we allow solutions to blow up at $\partial \Pi$. It has to be mentioned that, due to the structure of $\beta$ and the assumptions on $\nu$, the boundary splits into two regions: one which behaves as the interior of $\Pi$ and one where the solutions possibly blow up.

2. Multi-dimensional (semilinear and linear) integro-partial differential equations of the type (1.1) occur in the applications presented in Sections 3 and 4, which concern a class of backward stochastic differential equations in an unbounded jump Lévy setting. Backward 
stochastic differential equations (BSDEs henceforth), or more generally forward-backward stochastic differential equations, have received a lot of attention in recent years due to their many applications in stochastic control and mathematical finance. We refer to [20] for a general introduction to BSDEs. In [4], the authors consider a BSDE with terminal condition and coefficients being functions of a Lévy process. Under certain conditions they prove that the solution of the BSDE gives the unique viscosity solution to a system of semilinear integropartial differential equations set in $\mathbb{R}^{\mathrm{N}}$. In Section 3 we prove an existence and uniqueness result for a class of "Lévy driven" BSDEs having $(0, \infty)^{\mathrm{N}}$ as an invariant set. We then prove (under certain conditions) that the solution to the BSDE provides a unique viscosity solution of a semilinear integro-partial differential equation set in $(0, \infty)^{\mathrm{N}}$. Here uniqueness follows from the result in Section 2, which does not require a specification of a boundary condition. Compared to [4], the new features here are (i) the presence of an unbounded domain with nonsmooth boundary, (ii) the presence of a multidimensional Poisson random measure, (iii) a different structure for the jump amplitude and (iv) the treatment of the boundary. Finally, in Section 4 we provide new results on pricing European and American derivatives in rather general Lévy markets via BSDEs and linear integro-partial differential equations, relying on the results developed in Sections 2 and 3. For what concerns American derivatives we have to deal with an obstacle problem that is not directly included in the class described by (1.1)-(1.2). Nevertheless, it is well known that a comparison principle for obstacle problems can be easily derived from a corresponding principle for (1.1)-(1.2), see $[2,3,25]$. This argument will be detailed in Subsection 4.2. In a complete Black-Scholes market the unique arbitrage free price of any contingent claim is given in terms of an expectation value with respect to the unique equivalent martingale (risk neutral) measure, which in turn solves the Black-Scholes type partial differential equation. On the other hand, in an incomplete market there exist infinitely many equivalent martingale measures and corresponding arbitrage free prices. Consequently, to price a contingent claim one needs to select an appropriate equivalent martingale measure. Over the years several approaches for selecting an equivalent martingale measure have been suggested in the literature (see, e.g., $[10,29])$. Although Lévy markets are indeed incomplete, we are here not interested in any particular choice of an equivalent martingale measures. Instead we shall simply assume that we are given one, and then we derive, via the theory of BSDEs, a linear integro-partial differential equation satisfied by the corresponding arbitrage free price.

\section{Viscosity SOLUTIONS}

In this section we prove a comparison principle for viscosity solutions of (1.1)-(1.2). Let us start by introducing some notations:

$$
\begin{aligned}
& \Pi=(0, \infty)^{\mathrm{N}}, \\
& \bar{\Pi}=[0, \infty)^{\mathrm{N}} \text {, } \\
& \Gamma=\left\{x \in \partial \Pi: x_{i}>0 \text { as } i=1+\mathrm{N}^{\prime}, \cdots, \mathrm{N}\right\} \text {, } \\
& \tilde{\Pi}_{T}=\tilde{\Pi} \times[0, T), \\
& \mathcal{Q}(r)=[0, r]^{\mathrm{N}^{\prime}} \times[1 / r, r]^{\mathrm{N}-\mathrm{N}^{\prime}} . \\
& \Pi_{T}=\Pi \times[0, T), \\
& \bar{\Pi}_{T}=\bar{\Pi} \times[0, T], \\
& \tilde{\Pi}=\Pi \cup \Gamma \text {, } \\
& \tilde{\Pi}_{T}^{\star}=\tilde{\Pi} \times[0, T] \text {, }
\end{aligned}
$$

Here $\mathrm{N}^{\prime}$ stands for an integer between 0 and $\mathrm{N}$ that will be selected later on. In addition, for all $n>0$ we define a function on $\tilde{\Pi}$ by

$$
h_{n}(x)=\sum_{i=1}^{\mathrm{N}} x_{i}^{n}+\sum_{i=1+\mathrm{N}^{\prime}}^{\mathrm{N}} x_{i}^{-n},
$$

and we introduce the set

$$
\mathcal{P}_{n}(\Pi)=\left\{u: \Pi \rightarrow \mathbb{R}: \frac{|u(x)|}{1+h_{n}(x)} \text { is bounded }\right\},
$$


together with the norm $\|u\|_{n}=\sup _{x \in \Pi} \frac{|u(x)|}{1+h_{n}(x)}$. With a little abuse of notation, we set

$$
L^{\infty}\left(0, T ; \mathcal{P}_{n}(\Pi)\right)=\left\{u: \tilde{\Pi}_{T}^{\star} \rightarrow \mathbb{R}: \frac{|u(x, t)|}{1+h_{n}(x)} \text { is bounded }\right\} .
$$

In what follows, it will be useful to isolate the singularity of $\nu$ at the origin. Therefore we split $\mathcal{J} u$ into

$$
\mathcal{J}_{\kappa}(u, \varphi)(x, t)=\hat{\mathcal{J}}_{\kappa} \varphi(x, t)+\hat{\mathcal{J}}^{\kappa}(u, D \varphi(x, t))(x, t),
$$

where

$$
\begin{gathered}
E_{\kappa}=\left\{z \in \mathbb{R}^{2}: 0<|z|<\kappa\right\}, \quad E_{\kappa}^{c}=\mathbb{R}^{2} \backslash E_{\kappa}, \\
\hat{\mathcal{J}}_{\kappa} \varphi(x, t)=\int_{E_{\kappa}}[\varphi(x+\mathbf{x} \beta(x, t, z), t)-\varphi(x, t)-\beta(x, t, z) \cdot \mathbf{x} D \varphi(x, t)] \nu(d z), \\
\hat{\mathcal{J}}^{\kappa}(u, p)(x, t)=\int_{E_{\kappa}^{c}}[u(x+\mathbf{x} \beta(x, t, z), t)-u(x, t)-\beta(x, t, z) \cdot \mathbf{x} p] \nu(d z),
\end{gathered}
$$

We make the following assumptions on the integral operator $\mathcal{J}$ :

A.1 $\nu$ is a Radon measure on $E$ satisfying the integrability assumptions:

$$
\begin{gathered}
\int_{E}\left(1 \wedge|z|^{2}\right) \nu(d z)<\infty \\
\int_{E_{\kappa}^{c}} e^{\ell\left(z_{1}^{+}+\left|z_{2}\right|\right)} \nu(d z)<\infty
\end{gathered}
$$

for some given $\ell>0$ and for all $\kappa>0$.

Here and henceforth $z_{1}^{+}$and $z_{1}^{-}$stand for $\left(0 \vee z_{1}\right)=\max \left(0, z_{1}\right)$ and $\left(0 \vee-z_{1}\right)$, respectively.

A.2 $\beta \in \mathcal{C}\left(\bar{\Pi}_{T} \times E ; \mathbb{R}^{\mathbb{N}}\right)$ and there exist $n_{o} \geq 1$ such that

$$
e^{-\frac{\ell}{n_{o}}\left(z_{1}^{-}+\left|z_{2}\right|\right)} \leq \beta_{i}(x, t, z)+1 \leq e^{\frac{\ell}{n_{o}}\left(z_{1}^{+}+\left|z_{2}\right|\right)}
$$

for all $(x, t) \in \bar{\Pi}_{T}, z \in E$, and $i=1, \ldots$, N. Moreover for all $r>1$, there exists $\mathrm{C}_{r}>0$ such that

$$
\left|\beta(x, t, z)-\beta\left(x^{\prime}, t, z\right)\right| \leq \mathrm{C}_{r} e^{\ell\left(z_{1}^{+}+\left|z_{2}\right|\right)}\left|x-x^{\prime}\right|,
$$

for all $x, x^{\prime} \in \mathcal{Q}(r), t \in[0, T], z \in E$.

In force of assumption (A.2.i), $\beta$ behaves like $z$ near the origin. Thus the following lemma can be shown:

Lemma 2.1. Under assumptions A.1 and A.2, $\hat{\mathcal{J}}_{\kappa} \varphi(x, t)$ is finite for all $\varphi \in \mathcal{C}^{2,1}\left(\Pi_{T}\right)$, $(x, t) \in \Pi_{T}$, and $\kappa>0$. Moreover $\lim _{\kappa \rightarrow 0} \hat{\mathcal{J}}_{\kappa}(\varphi)(x, t)=0$.

However in many applications the solution in not $\mathcal{C}^{2}$, and consequently solutions have to be interpreted in the viscosity sense.

Let us mention that $\mathbf{x} \beta$ stands for the jump of the underlying Lévy process, so that (i) $\beta_{i} \sim+\infty$ means that the process is jumping towards infinity and (ii) $\beta_{i} \sim-1$ means that it is approaching the boundary $\partial \Pi$ at some point on the plane $\left\{x_{i}=0\right\}$. The structure condition (A.2.i) yields that (i) can happen if $z_{1} \sim+\infty$ or $z_{2} \sim \pm \infty$, while (ii) can happen if $z_{1} \sim-\infty$ or $z_{2} \sim \pm \infty$. Up to rearranging the order of the variables, we may assume without loss of generality that there exists an integer $\mathrm{N}^{\prime} \in\{0,1, \ldots, \mathrm{N}\}$ and $\mathrm{B}>0$ such that

$$
\beta_{i}(x, t, z)+1 \geq \mathrm{B}^{-\frac{\ell}{n_{o}}\left|z_{2}\right|} \quad \text { as } i=1+\mathrm{N}^{\prime}, \ldots, \mathrm{N},
$$

for all $(x, t) \in \bar{\Pi}_{T}$, and $z \in E$. In other words, the underlying process approaches the region $\partial \Pi \backslash \Gamma=\left\{x \in \partial \Pi: x_{i}=0\right.$ for some $\left.i=1+\mathrm{N}^{\prime}, \cdots, \mathrm{N}\right\}$ of the boundary only when $z_{2} \sim \pm \infty$, 
and not when $z_{1} \sim-\infty$. In force of assumption (A.1.ii), this matter is "unlikely", because the measure $\nu$ charges neighborhoods of $z_{2}= \pm \infty$ less than neighborhoods of $z_{1}=-\infty$. In some sense, the region $\partial \Pi \backslash \Gamma$ is further away than $\Gamma$, at least as far as infinity is concerned. This suggests that the points of $\partial \Pi \backslash \Gamma$ can be regarded as points at infinity, where the solutions are allowed to blow up. To be precise, the following lemma holds.

Lemma 2.2. Under assumptions A.1 and A.2, $\hat{\mathcal{J}}^{\kappa}(u, p)(x, t)$ is finite for any function $u \in L^{\infty}\left(0, T ; \mathcal{P}_{n_{o}}\left(\Pi_{T}\right)\right), p \in \mathbb{R}^{\mathbb{N}},(x, t) \in \Pi_{T}$, and $\kappa>0$.

Remark 2.3. It is easily seen that the conclusions of Lemmas 2.1 and 2.2 also hold for $x \in \tilde{\Pi}$, if $\varphi \in \mathcal{C}^{2,1}\left(\tilde{\Pi}_{T}\right)$ and $u$ is extended to $\tilde{\Pi}_{T}$ by giving any value between $\liminf _{\Pi \times[0, T) \ni(y, s) \rightarrow(x, t)} u(y, s)$ and $\limsup _{\Pi \times[0, T) \ni(y, s) \rightarrow(x, t)} u(y, s)$, respectively.

Both the limit situations $\mathrm{N}^{\prime}=\mathrm{N}$ and $\mathrm{N}^{\prime}=0$ may happen in applications.

Remark 2.4. Take a market composed by two assets which always jump in the same direction. They can be modelled by pure jump Lévy processes with $z \in \mathbb{R}, \beta_{i}(x, t, z)=e^{\eta_{i} z}-1$ and $\eta_{i}$ are given positive constants, $i=1,2$, see Section 3 . If, on the contrary, the two assets jump in opposite directions, then we must have $\eta_{1}>0$ and $\eta_{2}<0$. Both these matters are interesting in mathematical finance. The first one modelling perfect correlation between the assets, the second one modelling anti-correlation. They could be addressed in our framework by taking $\operatorname{supp} \nu$ as $\mathbb{R} \times\{0\}$ in the first case and as $\{0\} \times \mathbb{R}$ in the second case. Note that in any case assumption (A.1.ii) yields that the first momentum is finite.

Concerning the integro-differential equation (1.1), we suppose that $H$ is degenerate elliptic, satisfies some continuity properties and is monotone with respect to the non-local term $\mathcal{J} u$. Denote by $\mathcal{S}^{\mathrm{N}}$ the set of symmetric $\mathrm{N} \times \mathrm{N}$ real matrices. To be precise, we make then the following assumptions:

A.3 $H \in \mathcal{C}\left(\tilde{\Pi}_{T}^{\star} \times \mathbb{R} \times \mathbb{R}^{\mathbb{N}} \times \mathcal{S}^{\mathrm{N}} \times \mathbb{R}\right)$ and there exist a real number $\mathrm{L}$ and a modulus of continuity $\omega$ such that

$$
\begin{gathered}
H(x, t, u, p, M, J)-H\left(x, t, u^{\prime}, p, M, J\right) \geq \mathrm{L}\left(u-u^{\prime}\right) \quad \text { if } u \geq u^{\prime}, \\
\left|H(x, t, u, p, M, J)-H\left(x, t, u, p^{\prime}, M^{\prime}, J\right)\right| \leq \omega\left(\left|p-p^{\prime}\right|+\left\|M-M^{\prime}\right\|\right), \\
0 \geq H(x, t, u, p, M, J)-H\left(x, t, u, p, M, J^{\prime}\right) \geq-\omega\left(J-J^{\prime}\right) \quad \text { if } J \geq J^{\prime},
\end{gathered}
$$

for all $(x, t) \in \tilde{\Pi}_{T}^{\star}, u, u^{\prime}, J, J^{\prime} \in \mathbb{R}, p, p^{\prime} \in \mathbb{R}^{\mathrm{N}}$, and $M, M^{\prime} \in \mathcal{S}^{\mathrm{N}}$.

Moreover there is a family of moduli of continuity $\left(o_{r, R}\right)_{r>1, R>0}$ such that

$$
\begin{array}{r}
H\left(x, t, u, \frac{1}{\varepsilon} \mathbf{x}(x-y), \mathbf{x} M \mathbf{x}^{T}, J\right)- \\
H\left(y, t, u, \frac{1}{\varepsilon} \mathbf{y}(x-y), \mathbf{y} N \mathbf{y}^{T}, J\right) \\
\geq o_{r, R}\left(\frac{1}{\varepsilon}|x-y|^{2}+|x-y|\right),
\end{array}
$$

for all $x, y \in \mathcal{Q}(r), t \in[0, T], u \in[-R, R], J \in \mathbb{R}$ and $M, N \in \mathcal{S}^{\mathrm{N}}$ satisfying

$$
\left(\begin{array}{cc}
M & 0 \\
0 & -N
\end{array}\right) \leq \frac{3}{\varepsilon}\left(\begin{array}{cc}
I & -I \\
-I & I
\end{array}\right)
$$

Remark 2.5. All results can be trivially extended to the case $E=\mathbb{R}^{\mathrm{M}} \backslash\{0\}$ for an arbitrary integer M. In that case $z_{1}^{ \pm}$and $\left|z_{2}\right|$ have to be replaced by $\sum_{i=1}^{\mathrm{M}^{\prime}} z_{i}^{ \pm}$and $\sum_{i=1+\mathrm{M}^{\prime}}^{\mathrm{M}}\left|z_{i}\right|$, respectively.

We leave to the reader the extension to the case when $H$ also depends on an integral term of type (1.4). It suffices that (A.3.ii) holds also with respect to $\mathcal{I} u$ and that 
A.4 $\gamma \in \mathcal{C}\left(\bar{\Pi}_{T} \times E\right)$ satisfies

$$
0 \leq \gamma(x, t, z) \leq \mathrm{B}^{\prime}(1 \wedge|z|),
$$

for all $(x, t) \in \bar{\Pi}_{T}, z \in E$.Moreover for all $r>1$ there exist $\mathrm{C}_{r}^{\prime}>0$ such that

$$
\left|\gamma(x, t, z)-\gamma\left(x^{\prime}, t, z\right)\right| \leq \mathrm{C}_{r}^{\prime}\left(1 \wedge|z|^{2}\right)\left|x-x^{\prime}\right|,
$$

for all $x, x^{\prime} \in \mathcal{Q}(r), t \in[0, T], z \in E$.

Now we recall the notion of viscosity solutions for (1.1).

Definition 2.1. Let $\mathcal{O} \subset \bar{\Pi}$ contain $\Pi$, and set $\mathcal{O}_{T}=\mathcal{O} \times[0, T)$. If $u$ is any locally bounded function and $(x, t) \in \mathcal{O}_{T}$, we say that the inequality

$$
\left.-\partial_{t} u+H\left(x, t, u, \mathbf{x} D u, \mathbf{x} D^{2} u \mathbf{x}^{T}, \mathcal{J} u\right) \leq 0 \quad \text { (respectively, } \geq 0\right)
$$

holds true in the viscosity sense at $(x, t)$ (with respect to $\mathcal{O}_{T}$ ) if for any test function $\varphi \in$ $\mathcal{C}^{2,1}\left(\mathcal{O}_{T}\right) \cap \bigcup_{n<n_{o}} L^{\infty}\left(0, T ; \mathcal{P}_{n}(\Pi)\right)$ such that $u(x, t)=\varphi(x, t)$ and $(x, t)$ is a global maximum (respectively, minimum) point for $u-\varphi$ on $\mathcal{O}_{T}$, the inequality

$$
\left.-\partial_{t} \varphi+H\left(x, t, u, \mathbf{x} D \varphi, \mathbf{x} D^{2} \varphi \mathbf{x}^{T}, \mathcal{J}_{\kappa}(u, \varphi)\right) \leq 0 \quad \text { (respectively, } \geq 0\right)
$$

holds true in classical sense at $(x, t)$ for all $\kappa>0$.

A locally bounded function $u$ that is upper semicontinuous (respectively, lower semicontinuous) on $\Pi_{T}$ is a viscosity subsolution (respectively, viscosity supersolution) of (1.1) if

$$
-\partial_{t} u+H\left(x, t, u, \mathbf{x} D u, \mathbf{x} D^{2} u \mathbf{x}^{T}, \mathcal{J} u\right) \leq 0
$$

(respectively, $\geq 0$ ) holds true in the viscosity sense for all $(x, t) \in \Pi_{T}$ (with respect to $\left.\Pi_{T}\right)$. Any locally bounded function $u$ on $\Pi_{T}$ is a viscosity solution to (1.1) if its upper semicontinuous envelope is a viscosity subsolution and its lower semicontinuous envelope is a viscosity supersolution.

We refer to [11] for definitions of the upper and lower semicontinuous envelopes.

Remark 2.6. By making use of assumption (A.3.iii) and following [4, Lemma 3.4], it is clear that one may replace $\mathcal{J}_{\kappa}(u, \varphi)$ by $\mathcal{J} \varphi$ in the Definition 2.1. In the same way, one may replace global maximum (respectively, minimum) point by strict global maximum (respectively, minimum) point.

Two matters arise: (i) giving a suitable notion of terminal condition on $\partial \Pi \times\{T\}$ and (ii) dealing with the boundary $\partial \Pi_{T}$, where no data may feasibly be imposed. With respect to the notion of terminal condition, we choose here the naive one.

Definition 2.2. A locally bounded function u that is upper semicontinuous (respectively, lower semicontinuous) on $\Pi_{T}$ is a viscosity subsolution (respectively, a viscosity supersolution) to (1.1)-(1.2) if it is a viscosity subsolution (respectively, a viscosity supersolution) to (1.1) in the sense of Definition 2.1 and

$$
\begin{aligned}
& \limsup _{\Pi_{T} \ni(y, s) \rightarrow(x, T)} u(y, s) \leq \liminf _{\Pi \ni y \rightarrow x} u_{T}(y) \quad \text { in } \tilde{\Pi}, \\
& \text { (respectively, } \left.\quad \liminf _{\Pi_{T} \ni(y, s) \rightarrow(x, T)} u(y, s) \geq \limsup _{\Pi \ni y \rightarrow x} u_{T}(y) \quad \text { in } \tilde{\Pi}\right) .
\end{aligned}
$$

Any locally bounded function $u$ on $\Pi_{T}$ is a viscosity solution to (1.1)-(1.2) if its upper semicontinuous envelope is a viscosity subsolution and its lower semicontinuous envelope is a viscosity supersolution.

We devote the next subsection to an investigation of the behavior of sub- and supersolutions near the boundary of $\Pi_{T}$. 
2.1. Behavior at the boundary. The difficulty of dealing with a boundary where no data are assigned has been overcome in [6] by investigating constrained solutions. With respect to equations of the type (1.1) the use of constrained solutions is not necessary. In view of clarifying this issue, which shall play a central role in Theorem 2.10 , we recall a notion of constrained solution for (1.1).

Definition 2.3. A viscosity subsolution (respectively, viscosity supersolution) u to (1.1) is a constrained subsolution (respectively, a constrained supersolution) if it is upper semicontinuous (respectively, lower semicontinuous) on $\tilde{\Pi}_{T}$ and the viscosity inequality (2.1) holds true also at the points $(x, t) \in \Gamma \times[0, T)$. A constrained solution to (1.1) is any locally bounded function $u$ such that the upper and lower semicontinuous envelopes of $u$ are respectively constrained sub and-supersolutions to (1.1).

Notice that, if $\mathrm{N}^{\prime}=\mathrm{N}$, then $\tilde{\Pi}=\bar{\Pi}$ and our definition of constrained subsolution reduces to [6, Definition 4.1], up to the fact that we deal with possibly discontinuous solutions.

As we have mentioned before, the points in $\partial \Pi \backslash \Gamma$ have the character of being points at infinity. We will show that imposing a blow up rate is sufficient to pick up a unique solution. On the other hand, $\Gamma$ is made up by points that cannot be reached by the trajectories of the underlying process by drift motion (because of the structure $\mathbf{x} D u$ ), nor by diffusion (because of the structure $\mathbf{x} D^{2} u \mathbf{x}^{T}$ ), nor by jumping (because of the structure $\mathbf{x} \beta$ with $\left.\beta_{i}>-1\right)$. Inspired by a similar result in [5], we will establish that (1.1) holds also at $\Gamma$, up to boundary discontinuities, that is to say, any solution is constrained.

To this aim, for any upper semicontinuous function $u$ (respectively, lower semicontinuous function $v$ ) we set

$$
\begin{aligned}
& \tilde{u}(x, t)=\left\{\begin{array}{cc}
u(x, t) & \text { if }(x, t) \in \Pi \times[0, T), \\
\limsup _{\Pi_{T} \ni(y, s) \rightarrow(x, t)} u(y, s) & \text { if }(x, t) \in \Gamma \times[0, T), \\
v(x, t) & \text { if }(x, t) \in \Pi \times[0, T),
\end{array}\right. \\
& \tilde{v}(x, t)=\left\{\begin{array}{cc}
\liminf _{\Pi_{T} \ni(y, s) \rightarrow(x, t)} v(y, s) & \text { if }(x, t) \in \Gamma \times[0, T),
\end{array}\right.
\end{aligned}
$$

and then show some continuity of the non-local operators $\mathcal{I}$ and $\mathcal{J}$.

Lemma 2.7. Assume A.1-A.3. With $(x, t) \in \tilde{\Pi}_{T}$, let $\varphi \in \mathcal{C}^{2,1}\left(\Pi_{T}\right) \cap L^{\infty}\left(0, T ; \mathcal{P}_{n_{o}}(\Pi)\right)$ if $(x, t) \in \Pi_{T}$ and $\varphi \in \mathcal{C}^{2,1}\left(\tilde{\Pi}_{T}^{\star}\right) \cap L^{\infty}\left(0, T ; \mathcal{P}_{n_{o}}(\Pi)\right)$ if $(x, t) \in \Gamma \times[0, T)$. Let $u$ be an upper semicontinuous (respectively, a lower semicontinuous) function on $\Pi_{T}$ belonging to $L^{\infty}\left(0, T ; \mathcal{P}_{n_{o}}(\Pi)\right)$. Finally, let $\left(x_{n}, t_{n}\right)$ be a sequence in $\Pi_{T}$ such that $\left(x_{n}, t_{n}, u\left(x_{n}, t_{n}\right)\right) \rightarrow$ $(x, t, \tilde{u}(x, t))$. Then $\lim _{n \rightarrow \infty} \hat{\mathcal{J}}_{\kappa} \varphi\left(x_{n}, t_{n}\right)=\hat{\mathcal{J}}_{\kappa} \varphi(x, t), \limsup _{n \rightarrow \infty} \hat{\mathcal{J}}^{\kappa}(u, \varphi)\left(x_{n}, t_{n}\right) \leq \hat{\mathcal{J}}^{\kappa}(\tilde{u}, \varphi)(x, t)$, (respectively, $\left.\liminf _{n \rightarrow \infty} \hat{\mathcal{J}}^{\kappa}(u, \varphi)\left(x_{n}, t_{n}\right) \geq \hat{\mathcal{J}}^{\kappa}(\tilde{u}, \varphi)(x, t)\right)$, for any fixed $\kappa$.

Lemma 2.7 is an immediate consequence of Lebesgue's dominated convergence theorem, that may be applied thanks to assumptions A.1 and A.2.

Proposition 2.8. Let $u$ be an upper semicontinuous viscosity subsolution and $v$ a lower semicontinuous viscosity supersolution of (1.1), both belonging to $\bigcup_{n<n_{0}} L^{\infty}\left(0, T ; \mathcal{P}_{n}(\Pi)\right)$. Then the functions $\tilde{u}$ and $\tilde{v}$ defined in (2.2) and (2.3) are constrained sub and-supersolutions, respectively.

Proof. We carry out the proof only for the subsolution case, the supersolution case being completely analogous. Take $\left(x_{o}, t_{o}\right) \in \Gamma \times[0, T)$ and $\varphi \in \mathcal{C}^{2,1}\left(\tilde{\Pi}_{T}^{\star}\right) \bigcup_{n<n_{o}} L^{\infty}\left(0, T ; \mathcal{P}_{n}(\Pi)\right)$ such that $\varphi\left(x_{o}, t_{o}\right)=\tilde{u}\left(x_{o}, t_{o}\right)$ and $\left(x_{o}, t_{o}\right)$ is a strict global maximum point for $\tilde{u}-\varphi$. We 
want to perturb the test function $\varphi$ in order to achieve a sequence of maximum points in $\Pi_{T}$ converging to $\left(x_{o}, t_{o}\right)$. To this aim, we introduce the auxiliary functions $a, b \in \mathcal{C}^{2}(0, \infty)$

$$
\begin{aligned}
& a(\xi)= \begin{cases}-\log (\xi) & \text { if } \xi \in(0,1 / e], \\
\text { is decreasing and polynomial } & \text { if } \xi \in(1 / e, 1), \\
0 & \text { if } \xi \in[1, \infty),\end{cases} \\
& b(\xi)= \begin{cases}0 & \text { if } \xi \in(0,1], \\
\text { is increasing and polynomial } & \text { if } \xi \in(1,2), \\
\xi^{n_{o}} & \text { if } \xi \in[2, \infty),\end{cases}
\end{aligned}
$$

with the additional properties that $\left|\xi a^{\prime}(\xi)\right|,\left|\xi a^{\prime \prime}(\xi)\right| \leq \mathrm{A}_{o}$ and $\left|\xi b^{\prime}(\xi)\right|,\left|\xi b^{\prime \prime}(\xi)\right| \leq \mathrm{B}_{o} b(\xi)$. For any given $\alpha, \delta>0$ we then set

$$
\begin{gathered}
\mathbf{a}(x)=\sum_{i=1}^{\mathrm{N}^{\prime}} a\left(x_{i}\right), \quad \mathbf{b}_{\alpha}(x)=\sum_{i=1}^{\mathrm{N}} b\left(\frac{x_{i}}{\alpha}\right), \\
\varphi_{\alpha \delta}(x, t)=\varphi(x, t)+\mathbf{b}_{\alpha}(x)+\delta\left[\mathbf{a}(x)+h_{o}(x)\right], \\
\Psi_{\alpha \delta}(x, t)=\tilde{u}(x, t)-\varphi_{\alpha \delta}(x, t) .
\end{gathered}
$$

Here and in the following we write $h_{o}$ instead of $h_{n_{o}}$. We list in a lemma, to be proved later on, some relevant properties of $\Psi_{\alpha \delta}$.

Lemma 2.9. For any given $\alpha>1+2\left|x_{o}\right|$, there exists an infinitesimal sequence of parameters $\delta$ such that $\Psi_{\alpha \delta}$ achieves its global maximum at some point $\left(x_{\alpha \delta}, t_{\alpha \delta}\right) \in \Pi \times[0, T]$. In addition, up to an extracted subsequence,

$$
\begin{aligned}
\left(x_{\alpha \delta}, t_{\alpha \delta}\right) \rightarrow\left(x_{o}, t_{o}\right), & u\left(x_{\alpha \delta}, t_{\alpha \delta}\right) \rightarrow \tilde{u}\left(x_{o}, t_{o}\right) & \text { as } \delta & \rightarrow 0, \\
\delta \mathbf{a}\left(x_{\alpha \delta}\right) \rightarrow 0, & \delta h_{o}\left(x_{\alpha \delta}\right) \rightarrow 0, & \text { as } \delta & \rightarrow 0 .
\end{aligned}
$$

As a consequence, we may suppose that (i) for $\alpha$ large enough, $x_{\alpha \delta} \in[0, \alpha]^{\mathrm{N}}$ and $t_{\alpha \delta}<T$ for small $\delta$, and that (ii) $\left(x_{\alpha \delta}, t_{\alpha \delta}\right) \in \Pi_{T}$ is a global maximum point for $u-\varphi_{\alpha \delta}$ on $\Pi_{T}$. Therefore the viscosity inequality $(2.1)$ is satisfied at $\left(x_{\alpha \delta}, t_{\alpha \delta}\right)$ for any $\delta$ and $\alpha$.

In view of passing to the limit, we recall that for all $x, q \in \Pi$ we have

$$
\left|\mathbf{x} D h_{o}(x)\right|,\left|\mathbf{x} D^{2} h_{o}(x) \mathbf{x}^{T}\right| \leq \mathrm{C} h_{o}(x), \quad h_{o}(\mathbf{x} q) \leq h_{o}(x) h_{o}(q),
$$

where the parameter $\mathrm{C}$ does not depend on $(x, t)$.

By taking advantage of (2.4)-(2.6) and of assumption A.3, taking the limits with respect to $\delta \rightarrow 0$ and $\alpha \rightarrow \infty$ gives

$$
-\partial_{t} \varphi\left(x_{o}, t_{o}\right)+H\left(x_{o}, t_{o}, \tilde{u}, \mathbf{x}_{o} D \varphi, \mathbf{x}_{o} D^{2} \varphi \mathbf{x}_{o}^{T}, \limsup _{\substack{\delta \rightarrow 0 \\ \alpha \rightarrow \infty}} \mathcal{J}_{\kappa}\left(u, \varphi_{\alpha \delta}\right)\left(x_{\alpha \delta}, t_{\alpha \delta}\right)\right) \leq 0 .
$$

Therefore, in force of (A.3.iii), we may conclude by checking that

$$
\limsup _{\substack{\delta \rightarrow 0 \\ \alpha \rightarrow \infty}} \mathcal{J}_{\kappa}\left(u, \varphi_{\alpha \delta}\right)\left(x_{\alpha \delta}, t_{\alpha \delta}\right) \leq \mathcal{J}_{\kappa}(\tilde{u}, \varphi)\left(x_{o}, t_{o}\right) .
$$

After some computations we obtain

$$
\begin{gathered}
\mathcal{J}_{\kappa}\left(u, \varphi_{\alpha \delta}\right)\left(x_{\alpha \delta}, t_{\alpha \delta}\right) \leq \mathcal{J}_{\kappa}(u, \varphi)\left(x_{\alpha \delta}, t_{\alpha \delta}\right)+\mathrm{B} \int_{E_{\kappa}} \mathbf{b}_{\alpha}\left(\mathbf{x}_{\alpha \delta} \hat{q}_{\alpha \delta}(z)\right) \sum_{i=1}^{\mathrm{N}} \frac{\beta_{i}\left(x_{\alpha \delta}, t_{\alpha \delta}, z\right)^{2}}{\hat{q}_{\alpha \delta i}(z)^{2}} \nu(d z) \\
+\delta\left(\mathrm{A}+\mathrm{C} h_{o}\left(x_{\alpha \delta}\right)\right)\left[\int_{E_{\kappa}^{c}}\left|\beta\left(x_{\alpha \delta}, t_{\alpha \delta}, z\right)\right| \nu(d z)+\int_{E_{\kappa}} h_{o}\left(\hat{q}_{\alpha \delta}(z)\right) \sum_{i=1}^{\mathrm{N}} \frac{\beta_{i}\left(x_{\alpha \delta}, t_{\alpha \delta}, z\right)^{2}}{\hat{q}_{\alpha \delta i}(z)^{2}} \nu(d z)\right],
\end{gathered}
$$


where $\hat{q}_{\alpha \delta}(z)$ stands for a suitable point in the segment line joining the points $(1, \cdots, 1) \in \mathbb{R}^{N}$ and $\left(1+\beta_{1}\left(x_{\alpha \delta}, t_{\alpha \delta}, z\right), \cdots, 1+\beta_{\mathrm{N}}\left(x_{\alpha \delta}, t_{\alpha \delta}, z\right)\right) \in \mathbb{R}^{\mathrm{N}}$. Keeping in mind A.2, taking the limit with respect to $\delta$ and applying Lemma 2.7 gives

$$
\limsup _{\delta \rightarrow 0} \mathcal{J}_{\kappa}\left(u, \varphi_{\alpha \delta}\right)\left(x_{\alpha \delta}, t_{\alpha \delta}\right) \leq \mathcal{J}_{\kappa}(u, \varphi)\left(x_{o}, t_{o}\right)+\mathrm{C} \max _{Q_{\kappa}} \mathbf{b}_{\alpha} \int_{E_{\kappa}}|z|^{2} \nu(d z),
$$

where the parameter C does not depend by $\alpha$, and $Q_{\kappa}=\left[0,2\left|x_{o}\right|\left(1+e^{\frac{\ell}{n_{o}} \kappa}\right)\right]^{\mathrm{N}}$. Eventually we may conclude by checking that $\max _{Q_{\kappa}} \mathbf{b}_{\alpha} \rightarrow 0$ as $\alpha \rightarrow \infty$, for any given $\kappa>0$. But this is trivial because $\mathbf{b}_{\alpha} \equiv 0$ on $[0, \alpha]^{\mathrm{N}}$ by construction.

Proof of Lemma 2.9. Take $\alpha>1+2\left|x_{o}\right|$ and set $M_{\alpha \delta}=\sup \left\{\tilde{u}(x, t)-\varphi_{\alpha \delta}(x, t)\right\}$. Notice that $M_{\alpha \delta} \leq \max \{\tilde{u}-\varphi\}=0$. Next, take a maximizing sequence $\left(x_{j}, t_{j}\right) \in \Pi_{T}$ such that $\left(x_{j}, t_{j}, u\left(x_{j}, t_{j}\right)-\varphi\left(x_{j}, t_{j}\right)\right) \rightarrow\left(x_{o}, t_{o}, 0\right)$ and choose an infinitesimal sequence $\delta_{j}>0$ in such a way that $\delta_{j}\left[\mathbf{a}\left(x_{j}\right)+h_{o}\left(x_{j}\right)\right] \leq 1 / j$. Without loss of generality, we may suppose that $x_{j} \in\left[0,1+2\left|x_{o}\right|\right]^{\mathrm{N}}$ for all $j$, so that $\mathbf{b}_{\alpha}\left(x_{j}\right)=0$ and therefore $M_{\alpha \delta_{j}} \rightarrow 0$ as $j \rightarrow \infty$. On the other hand, there exists $n<n_{o}$ such that $\tilde{u}-\varphi \in L^{\infty}\left(0, T ; \mathcal{P}_{n}(\Pi)\right)$. Denoting $\mathrm{C}=\sup _{t \in[0, T)}\|\tilde{u}(\cdot, t)-\varphi(\cdot, t)\|_{n}$ we have

$$
\Psi_{\delta_{j}}(x, t) \leq \sum_{i=1}^{\mathrm{N}}\left(\mathrm{C}-\delta_{j} x_{j}{ }_{i}^{n_{o}-n}\right) x_{j}^{n}+\sum_{i=1+\mathrm{N}^{\prime}}^{\mathrm{N}}\left(\mathrm{C}-\delta_{j} x_{j}{ }^{-n_{o}+n}\right) x_{j}{ }^{-n} \rightarrow-\infty,
$$

if $|x| \rightarrow \infty$ or $x \rightarrow \bar{x} \in \partial \Pi \backslash \Gamma$. Hence standard semicontinuity arguments give that $\Psi_{\alpha \delta_{j}}$ has a maximum point $\left(x_{\alpha \delta_{j}}, t_{\alpha \delta_{j}}\right) \in \tilde{\Pi} \times[0, T]$. Moreover, $\Psi_{\alpha \delta_{j}}\left(x_{\alpha \delta_{j}}, t_{\alpha \delta_{j}}\right) \rightarrow 0$ as $j \rightarrow \infty$. As a consequence

$$
\lim _{j \rightarrow \infty}\left\{\delta_{j}\left[\mathbf{a}\left(x_{\alpha \delta_{j}}\right)+h_{o}\left(x_{\alpha \delta_{j}}\right)\right]+\mathbf{b}_{\alpha}\left(x_{\alpha \delta_{j}}\right)\right\}=\lim _{j \rightarrow \infty}\left[\tilde{u}\left(x_{\alpha \delta_{j}}, t_{\alpha \delta_{j}}\right)-\varphi\left(x_{\alpha \delta_{j}}, t_{\alpha \delta_{j}}\right)\right] .
$$

Because the limit appearing in the right-hand side is less than or equal to zero by construction, (2.5) is established. In addition, $\lim _{j \rightarrow \infty} \mathbf{b}_{\alpha}\left(x_{\alpha \delta_{j}}\right)=0$ allows us to suppose that $x_{\alpha \delta_{j}}$ is bounded uniformly with respect to $j$ (for fixed $\alpha$ ). As a consequence, up to an extracted subsequence, $\left(x_{\alpha \delta_{j}}, t_{\alpha \delta_{j}}\right)$ tends to some $(\hat{x}, \hat{t}) \in \tilde{\Pi} \times[0, T]$, so that standard semicontinuity arguments give (2.4).

2.2. Comparison Principle. We now prove a comparison result between (semicontinuous) viscosity sub- and supersolutions which satisfy a suitable growth condition near $\partial \Pi$ and for large $x$.

Theorem 2.10. Assume A.1-A.3 and let $u, v \in \bigcup_{n<n_{o}} L^{\infty}\left(0, T ; \mathcal{P}_{n}(\Pi)\right)$ be respectively an upper semicontinuous viscosity subsolution and a lower semicontinuous viscosity supersolution to (1.1) such that

$$
\limsup _{\Pi_{T} \ni(y, s) \rightarrow(x, T)} u(y, s) \leq \liminf _{\Pi_{T} \ni(y, s) \rightarrow(x, T)} v(y, s) \quad \text { for all } x \in \tilde{\Pi} .
$$

Then $u \leq v$ on $\Pi \times[0, T]$. Moreover, the functions $\tilde{u}$ and $\tilde{v}$ defined in (2.2) and (2.3) satisfy $\tilde{u} \leq \tilde{v}$ on $\tilde{\Pi} \times[0, T]$.

Before proving Theorem 2.10, we explicitly state an immediate consequence of it.

Corollary 2.11. Assume A.1-A.3 and take $u_{T} \in \mathcal{C}(\tilde{\Pi}) \cap \mathcal{P}_{n}(\Pi)$ for some $n<n_{o}$. Let $u \in \bigcup_{n<n_{o}} L^{\infty}\left(0, T ; \mathcal{P}_{n}(\Pi)\right)$ be a (possibly discontinuous) viscosity solution to (1.1)-(1.2).

Then $u$ is the unique viscosity solution in the class $\bigcup_{n<n_{0}} L^{\infty}\left(0, T ; \mathcal{P}_{n}(\Pi)\right)$. Moreover it is 
continuous on $\Pi_{T}$ and can be extended continuously to $\tilde{\Pi} \times[0, T]$ by setting

$$
\mathbf{u}(x, t)=\left\{\begin{array}{cl}
u(x, t) & \text { if }(x, t) \in \Pi_{T}, \\
\lim _{\Pi_{T} \ni(y, s) \rightarrow(x, t)} u(y, s) & \text { if }(x, t) \in \Gamma \times[0, T] \cup \Pi \times\{T\} .
\end{array}\right.
$$

The function $\mathbf{u}$ still solves (1.1)-(1.2), and satisfies $\mathbf{u}(x, T)=u_{T}(x)$ for all $x \in \tilde{\Pi}$.

Let us now give the proof of the comparison principle.

Proof of Theorem 2.10. Before entering into the details of the proof, we recall that we may assume that the parameter L appearing in hypothesis (A.3.i) is equal to 1 (if not, we simply multiply $u$ and $v$ by $\exp ((\mathrm{L}-1)(T-t)))$.

The theorem follows if we can show that $\tilde{u} \leq \tilde{v}$ on $\tilde{\Pi} \times[0, T]$. We argue by contradiction, and suppose that

$$
\mathcal{M}=\sup \{\tilde{u}(x, t)-\tilde{v}(x, t):(x, t) \in \tilde{\Pi} \times[0, T]\}>0 .
$$

Because it is not known a priori whether $\mathcal{M}$ is finite or not, we approximate $\tilde{u}$ and $\tilde{v}$ by

$$
\tilde{u}_{\delta}(x, t)=\tilde{u}(x, t)-\delta h_{o}(x), \quad \tilde{v}_{\delta}(x, t)=\tilde{v}(x, t)+\delta h_{o}(x),
$$

and we look at the upper semicontinuous function

$$
\Psi_{\delta}(x, t)=\tilde{u}_{\delta}(x, t)-\tilde{v}_{\delta}(x, t), \quad(x, t) \in \tilde{\Pi} \times[0, T] .
$$

Arguing as in the proof of Lemma 2.9, one may easily check that there exists an infinitesimal sequence of parameters $\delta$ (that we still denote by $\delta$ ) such that $\Psi_{\delta}$ has a maximum point $\left(x_{\delta}, t_{\delta}\right) \in \tilde{\Pi} \times[0, T]$. Moreover

$$
\tilde{u}\left(x_{\delta}, t_{\delta}\right)-\tilde{v}\left(x_{\delta}, t_{\delta}\right) \rightarrow \mathcal{M}, \quad \delta h_{o}\left(x_{\delta}\right) \rightarrow 0,
$$

as $\delta \rightarrow 0$.

Notice that our assumption $\mathcal{M}>0$ allows us to suppose that $\tilde{u}\left(x_{\delta}, t_{\delta}\right)-\tilde{v}\left(x_{\delta}, t_{\delta}\right)>0$ for all $\delta$ and therefore, recalling hypothesis (2.7), that $t_{\delta}<T$ for all $\delta$. Besides $\tilde{u}_{\delta}$ and $\tilde{v}_{\delta}$ satisfy in the viscosity sense two modified integro-partial differential inequalities.

Lemma 2.12. There exists $\mathrm{D} \geq 0$ not depending on $\delta$ such that $\tilde{u}_{\delta}$ and $\tilde{v}_{\delta}$ are respectively a constrained subsolution to

$$
-\partial_{t} \tilde{u}_{\delta}+H\left(x, t, \tilde{u}_{\delta}, \mathbf{x} D \tilde{u}_{\delta}, \mathbf{x} D^{2} \tilde{u}_{\delta} \mathbf{x}^{T}, \mathcal{J} \tilde{u}_{\delta}\right)=-\delta h_{o}(x)+\omega\left(\mathrm{D} \delta h_{o}(x)\right),
$$

and a constrained supersolution to

$$
-\partial_{t} \tilde{v}_{\delta}+H\left(x, t, \tilde{v}_{\delta}, \mathbf{x} D \tilde{v}_{\delta}, \mathbf{x} D^{2} \tilde{v}_{\delta} \mathbf{x}^{T}, \mathcal{J} \tilde{v}_{\delta}\right)=\delta h_{o}(x)-\omega\left(\mathrm{D} \delta h_{o}(x)\right),
$$

for all $\delta>0$.

We shall later on give a sketch of the proof of this statement. For now we prefer to show how to use these integro-partial differential inequalities to get a contradiction. To this aim, we double the $x$-variable by considering the function

$$
\tilde{\Pi}^{2} \times[0, T] \ni(x, y, t) \mapsto \tilde{u}_{\delta}(x, t)-\tilde{v}_{\delta}(y, t)-\psi(x, y),
$$

where the penalization function $\psi(x, y)$ is defined by

$$
\psi(x, y)=\frac{1}{2 \varepsilon}|x-y|^{2} .
$$

By a classical argument in the theory of viscosity solutions, for any fixed $\delta, \varepsilon>0$ this function has a maximum point $\left(x_{\delta}^{\varepsilon}, y_{\delta}^{\varepsilon}, t_{\delta}^{\varepsilon}\right) \in \tilde{\Pi}^{2} \times[0, T]$ which satisfies

$$
\begin{gathered}
x_{\delta}^{\varepsilon}, y_{\delta}^{\varepsilon} \rightarrow x_{\delta}, \quad t_{\delta}^{\varepsilon} \rightarrow t_{\delta}, \quad \frac{1}{2 \varepsilon}\left|x_{\delta}^{\varepsilon}-y_{\delta}^{\varepsilon}\right|^{2} \rightarrow 0, \\
\tilde{u}_{\delta}\left(x_{\delta}^{\varepsilon}, t_{\delta}^{\varepsilon}\right)-\tilde{v}_{\delta}\left(y_{\delta}^{\varepsilon}, t_{\delta}^{\varepsilon}\right) \rightarrow \tilde{u}_{\delta}\left(x_{\delta}, t_{\delta}\right)-\tilde{v}_{\delta}\left(y_{\delta}, t_{\delta}\right),
\end{gathered}
$$


as $\varepsilon \rightarrow 0$. We remark that (2.12), together with (2.8), allows us to suppose without loss of generality that for all $\delta, \varepsilon>0$

$$
\begin{array}{cc}
x_{\delta}^{\varepsilon}, y_{\delta}^{\varepsilon} \in \mathcal{Q}\left(r_{\delta}\right), & t_{\delta}^{\varepsilon}<T, \\
\left|u_{\delta}\left(x_{\delta}^{\varepsilon}, t_{\delta}^{\varepsilon}\right)\right|,\left|v_{\delta}\left(y_{\delta}^{\varepsilon}, t_{\delta}^{\varepsilon}\right)\right| \leq R_{\delta}, & \tilde{u}_{\delta}\left(x_{\delta}^{\varepsilon}, t_{\delta}^{\varepsilon}\right)-\tilde{v}_{\delta}\left(y_{\delta}^{\varepsilon}, t_{\delta}^{\varepsilon}\right)>0,
\end{array}
$$

where $r_{\delta}>1$ and $R_{\delta}>0$ do not depend on $\varepsilon$.

To simplify notations in what follows, we replace the subscripts $\delta, \varepsilon$ by over-bars and we omit the time dependence.

It follows from [16] that there exist $\bar{\tau} \in \mathbb{R}$ and two symmetric matrices $\bar{M}$ and $\bar{N}$ such that

$$
\left(\begin{array}{cc}
\bar{M} & 0 \\
0 & -\bar{N}
\end{array}\right) \leq \frac{3}{\varepsilon}\left(\begin{array}{cc}
I & -I \\
-I & I
\end{array}\right)
$$

and, since $\tilde{u}_{\delta}, \tilde{v}_{\delta}$ solve (2.9) and (2.10) respectively,

$$
\begin{array}{r}
-\bar{\tau}+H\left(\bar{x}, \tilde{u}_{\delta}, \overline{\mathbf{x}} p, \overline{\mathbf{x}} \bar{M} \overline{\mathbf{x}}^{T}, \hat{\mathcal{J}}_{\kappa} \psi(\bar{x}, \bar{y})+\hat{\mathcal{J}}^{\kappa}\left(\tilde{u}_{\delta}, \bar{p}\right)(\bar{x})\right) \leq-\delta h_{o}(\bar{x})+2 \omega\left(\mathrm{D} \delta h_{o}(\bar{x})\right), \\
-\bar{\tau}+H\left(\bar{y}, \tilde{v}(\bar{y}), \overline{\mathbf{y}} \bar{p}, \overline{\mathbf{y}} \bar{N} \overline{\mathbf{y}}^{T},-\hat{\mathcal{J}}_{\kappa} \psi(\bar{x}, \bar{y})+\hat{\mathcal{J}}^{\kappa}\left(\tilde{v}_{\delta}, \bar{p}\right)(\bar{y})\right) \geq \delta h_{o}(\bar{y})-2 \omega\left(\mathrm{D} \delta h_{o}(\bar{y})\right),
\end{array}
$$

where $\bar{p}=\frac{1}{\varepsilon}(\bar{x}-\bar{y})$. Subtracting the two inequalities yields

$$
\bar{H}_{\kappa} \leq \bar{G},
$$

where

$$
\begin{aligned}
\bar{H}_{\kappa}:= & H\left(\bar{x}, \tilde{u}_{\delta}(\bar{x}), \overline{\mathbf{x}} p, \overline{\mathbf{x}} \bar{M} \overline{\mathbf{x}}^{T}, \hat{\mathcal{J}}_{\kappa} \psi(\bar{x}, \bar{y})+\hat{\mathcal{J}}^{\kappa}\left(\tilde{u}_{\delta}, \bar{p}\right)(\bar{x})\right) \\
& -H\left(\bar{y}, \tilde{v}(\bar{y}), \overline{\mathbf{y}} \bar{p}, \overline{\mathbf{y}} \bar{N} \overline{\mathbf{y}}^{T},-\hat{\mathcal{J}}_{\kappa} \psi(\bar{x}, \bar{y})+\hat{\mathcal{J}}^{\kappa}\left(\tilde{v}_{\delta}, \bar{p}\right)(\bar{y})\right), \\
\bar{G}:= & -\delta\left[h_{o}(\bar{x})+h_{o}(\bar{y})\right]+\omega\left(\mathrm{D} \delta h_{o}(\bar{x})\right)+\omega\left(\mathrm{D} \delta h_{o}(\bar{y})\right) .
\end{aligned}
$$

Concerning $\bar{G},(2.8)$ and (2.12) imply that $\lim _{\delta \rightarrow 0} \lim _{\varepsilon \rightarrow 0} \bar{G}=0$. Concerning $\bar{H}_{\kappa}$, by making use of hypothesis A.3 and remembering (2.13), (2.14) we obtain

$$
\bar{H}_{\kappa} \geq\left[u_{\delta}(\bar{x})-v_{\delta}(\bar{y})\right]+\omega\left(\left(\bar{J}_{\kappa}\right)^{+}\right)-o_{r_{\delta}, R_{\delta}}\left(\frac{1}{\varepsilon}|\bar{x}-\bar{y}|^{2}+|\bar{x}-\bar{y}|\right)
$$

where we have used the short-hand notation

$$
\bar{J}_{\kappa}:=2 \hat{\mathcal{J}}_{\kappa} \psi(\bar{x}, \bar{y})+\hat{\mathcal{J}}^{\kappa}\left(\tilde{u}_{\delta}, \bar{p}\right)(\bar{x})-\hat{\mathcal{J}}^{\kappa}\left(\tilde{v}_{\delta}, \bar{p}\right)(\bar{y}) .
$$

Now (2.12) implies that the third term in the right-hand side of (2.16) tends to zero as $\varepsilon \rightarrow 0$. Regarding the estimation of the second term, we recall that $\hat{\mathcal{J}}_{\kappa} \psi(\bar{x}, \bar{y})$ tends to zero as $\kappa \rightarrow 0$ for any fixed value of $\varepsilon$, by Lemma 2.1. Hence we only need to estimate the positive part of

$$
\hat{J}_{\kappa}:=\hat{\mathcal{J}}^{\kappa}\left(\tilde{u}_{\delta}, \bar{p}\right)(\bar{x})-\hat{\mathcal{J}}^{\kappa}\left(\tilde{v}_{\delta}, \bar{p}\right)(\bar{y}),
$$

uniformly with respect to $\kappa \in(0,1)$. To this end, we split the integral into the two sets $E_{1}^{c}$, $A_{\kappa}=\{\kappa<|z| \leq 1\}$ and we write

$$
\begin{aligned}
\hat{J}_{\kappa}= & \hat{\mathcal{J}}^{1}\left(\tilde{u}_{\delta}, \bar{p}\right)(\bar{x})-\hat{\mathcal{J}}^{1}\left(\tilde{v}_{\delta}, \bar{p}\right)(\bar{y}) \\
& +\int_{A_{\kappa}}\left[\left[\tilde{u}_{\delta}(\hat{x})-\tilde{v}_{\delta}(\hat{y})\right]-\left[\tilde{u}_{\delta}(\bar{x})-\tilde{v}_{\delta}(\bar{y})\right]-\frac{1}{\varepsilon}(\overline{\mathbf{x}} \beta(\bar{x}, z)-\overline{\mathbf{y}} \beta(\bar{y}, z)) \cdot(\bar{x}-\bar{y})\right] \nu(d z)
\end{aligned}
$$

where we have used the shortened notations $\hat{x}=\bar{x}+\overline{\mathbf{x}} \beta(\bar{x}, z), \hat{y}=\bar{y}+\overline{\mathbf{y}} \beta(\bar{y}, z)$. 
With respect to the first term, we notice that

$$
\begin{gathered}
\hat{\mathcal{J}}^{1}\left(\tilde{u}_{\delta}, \bar{p}\right)(\bar{x})-\hat{\mathcal{J}}^{1}\left(\tilde{v}_{\delta}, \bar{p}\right)(\bar{y}) \leq \\
\int_{E_{1}^{c}}\left[\left[\tilde{u}_{\delta}(\hat{x})-\tilde{v}_{\delta}(\hat{y})\right]-\left[\tilde{u}_{\delta}(\bar{x})-\tilde{v}_{\delta}(\bar{y})\right]\right] \nu(d z)+\int_{E_{1}^{c}} \frac{1}{\varepsilon}|\bar{x}-\bar{y}||\overline{\mathbf{x}} \beta(\bar{x}, z)-\overline{\mathbf{y}} \beta(\bar{y}, z)| \nu(d z) .
\end{gathered}
$$

Assumptions A.1 and A.2 allow us to apply Lebesgue's dominated convergence theorem to both the integrals. Therefore (2.12) gives

$$
\limsup _{\varepsilon \rightarrow 0}\left[\hat{\mathcal{J}}^{1}\left(\tilde{u}_{\delta}, \bar{p}\right)(\bar{x})-\hat{\mathcal{J}}^{1}\left(\tilde{v}_{\delta}, \bar{p}\right)(\bar{y})\right] \leq \int_{E_{1}^{\mathrm{c}}}\left[\Psi_{\delta}\left(\hat{x}_{\delta}, t_{\delta}\right)-\Psi_{\delta}\left(x_{\delta}, t_{\delta}\right)\right] \nu(d z) \leq 0,
$$

because $\left(x_{\delta}, t_{\delta}\right)$ is a maximum point for $\Psi_{\delta}$. On the other hand, arguing as in the proof of [6, Theorem 4.2], one obtains that also the second term is bounded from above by some infinitesimal quantity (with respect to $\varepsilon$ ) that does not depend on $\kappa$. By plugging the obtained estimates in (2.16) and making use of (2.8) we obtain $\lim _{\delta \rightarrow 0} \lim _{\varepsilon \rightarrow 0} \lim _{\kappa \rightarrow 0} \bar{H}_{\kappa} \geq \mathcal{M}$. Eventually extracting the limit with respect to $\kappa, \varepsilon$ and $\delta$ in (2.15) gives the contradiction $\mathcal{M} \leq 0$.

This section is concluded by checking the validity of Lemma 2.12 .

Proof of Lemma 2.12. We give the proof only for (2.9), the proof of (2.10) being similar. By construction if $\varphi$ is a test function for $\tilde{u}_{\delta}$, then $\varphi_{\delta}=\varphi+\delta h_{o}$ is a test function for $\tilde{u}$. In particular for all $\kappa>0$ we have $\mathcal{J}_{\kappa}\left(\tilde{u}_{\delta}, \varphi\right)=\mathcal{J}_{\kappa}\left(\tilde{u}, \varphi_{\delta}\right)-\delta \mathcal{J}\left(h_{o}\right)$. Therefore, keeping in mind that $\tilde{u}$ is a constrained subsolution to (1.1), hypothesis A.3 and the estimates (2.6) give

$$
\begin{gathered}
-\partial_{t} \tilde{u}_{\delta}+H\left(x, t, \tilde{u}_{\delta}, \mathbf{x} D \varphi, \mathbf{x} D^{2} \varphi \mathbf{x}^{T}, \mathcal{J}_{\kappa}\left(\tilde{u}_{\delta}, \varphi\right)\right) \leq \\
-\delta h_{o}(x)+\omega\left(2 \delta \mathrm{c} h_{o}(x)\right)+\omega\left(\delta\left(\mathcal{J} h_{o}(x)\right)^{+}\right),
\end{gathered}
$$

for all $\kappa>0$ and $(x, t) \in \tilde{\Pi}_{T}$. The thesis follows after computations similar to the ones in Proposition 2.8.

\section{Backward Stochastic Differential Equations}

This section is devoted to extending the results in [4]. More precisely, we present here an existence and uniqueness result for a class of BSDEs in a rather general Lévy setting. Moreover, we show how to relate, via viscosity solutions, this stochastic problem to a semilinear integro-partial differential equation on a domain with a boundary, however without specifying a boundary condition.

Let us consider a stochastic N-dimensional process $X_{t}$ defined by means of its dynamics

$$
d X_{t}=\mathbf{X}_{t}\left[b\left(X_{t}, t\right) d t+\sigma\left(X_{t}, t\right) d W_{t}+\int_{E} \beta\left(X_{t^{-}}, t, z\right) \tilde{N}(d t, d z)\right]
$$

where $\mathbf{X}_{t}=\operatorname{diag}\left(X_{t}^{1}, \ldots, X_{t}^{\mathrm{N}}\right)$. Here $W_{t}$ is a M-dimensional standard Brownian motion, $1 \leq \mathrm{M} \leq \mathrm{N}, \tilde{N}$ is the compensated martingale measure of a $\mathrm{N}$-dimensional Poisson random measure $N$ defined on $\mathbb{R}^{+} \times E$ with compensator $\lambda(d t, d z)=d t \times \nu(d z), \nu(d z)$ is its Lévy measure, and $\nu: \mathcal{B}(E) \rightarrow \mathbb{R}^{\mathrm{N}}$,

$$
\nu(d z)=\left(\nu^{1}(d z), \nu^{2}(d z), \ldots, \nu^{\mathrm{N}}(d z)\right)
$$

is the N-dimensional Lévy measure. Here $b: \bar{\Pi}_{T} \rightarrow \mathbb{R}^{\mathrm{N}}, \sigma: \bar{\Pi}_{T} \rightarrow \mathbb{R}^{\mathrm{N} \times \mathrm{M}}, \beta: \bar{\Pi}_{T} \times E \rightarrow \mathbb{R}^{\mathrm{N} \times \mathrm{N}}$.

The $X_{t}$ process is considered in the setting of a stochastic basis $\left(\Omega, \mathcal{F},\left(\mathcal{F}_{t}\right)_{t \geq 0}, \mathcal{P}\right)$ such that $\mathcal{F}_{0}$ contains all $\mathcal{P}$-null elements of $\mathcal{F}$, and $\mathcal{F}_{t^{+}}=\bigcap_{\varepsilon>0} \mathcal{F}_{t+\varepsilon}=\mathcal{F}_{t}, t>0$. We suppose that the filtration is generated by the two mutually independent processes $W_{t}$ and $N(d t, d z)$.

We will assume that the measure $\nu^{j}(d z)$ satisfies assumption (A.1) and that $\beta_{j}$ satisfies assumption (A.2), for all $j=1, \ldots, \mathrm{N}$, and that 
(B.1) $b: \bar{\Pi}_{T} \rightarrow \mathbb{R}^{\mathrm{N}}$ and $\sigma: \bar{\Pi}_{T} \rightarrow \mathbb{R}^{\mathrm{N} \times \mathrm{M}}$ are bounded and globally Lipschitz continuous with respect to $x$, namely that for all $x, y \in \Pi$ there exists a positive constant $\mathrm{C}$ such that

$$
\begin{aligned}
& |b(x, t)-b(y, t)|+|\sigma(x, t)-\sigma(y, t)| \leq \mathrm{C}|x-y|, \\
& |b(x, t)|+|\sigma(x, t)| \leq \mathrm{C} .
\end{aligned}
$$

We do not make any assumptions on the rank of the matrices $\sigma$ and $\beta$.

Under the assumptions (A.1), (A.2) and (B.1) on the coefficients, in [13] it is proved that there exists a unique solution of problem (3.1) with initial data $X_{0}=x_{o} \in \Pi$, which is denoted by $\left\{X_{t}^{0}\left(x_{o}\right)\right\}_{t \geq 0}$.

Lemma 3.1. Let us suppose that assumptions (A.1), (A.2) and (B.1) hold. Then the solution $X_{s}^{t_{o}}(x)$ of (3.1) with initial data $X_{t_{o}}^{t_{o}}(x) \in \Pi$ verifies $X_{s}^{t_{o}}(x) \in \Pi$ a.s. for all $s \geq t_{o} \geq 0$.

Proof. By the assumptions on the coefficients, the solution of the $i$ th component of equation (3.1) exists and it is unique in probability [17]. By Ito's formula, omitting the dependence of the coefficients on $\left(X_{t}, t\right)$,

$$
\begin{aligned}
d\left(\log X_{t}^{i}\right)= & {\left[b^{i}+\sum_{j=1}^{\mathrm{N}}\left(\ln \left(1+\beta_{j}^{i}(z)\right)-\beta_{j}^{i}(z)\right) \nu^{j}(d z)-\frac{1}{2} \sum_{j=1}^{\mathrm{M}}{\sigma_{j}^{i}}^{2}\right] d t } \\
& +\sum_{j=1}^{\mathrm{M}} \sigma_{j}^{i} d W_{t}^{j}+\sum_{j=1}^{\mathrm{N}} \int_{E} \ln \left(1+\beta_{j}^{i}(z)\right) \tilde{N}^{j}(d t, d z) .
\end{aligned}
$$

Integrating over $[t, s]$ and taking the exponential we get

$$
\begin{gathered}
\left(X_{s}^{t_{o}}\right)^{i}(x)=X_{t_{o}}^{t_{o}} \exp \left\{\int_{t_{o}}^{s}\left[b^{i}-\frac{1}{2} \sum_{j=1}^{\mathrm{M}}{\sigma_{j}^{i}}^{2}+\sum_{j=1}^{\mathrm{N}} \int_{E}\left(\log \left(1+\beta_{j}^{i}\left(\tau, X_{\tau}, z\right)\right)-\beta_{j}^{i}(z)\right) \nu^{j}(d z)\right] d \tau\right. \\
\left.+\sum_{j=1}^{\mathrm{M}} \sigma_{j}^{i} d W_{\tau}^{j}+\sum_{j=1}^{\mathrm{N}} \int_{t}^{s} \int_{E}\left(\log \left(1+\beta_{j}^{i}(z)\right)-\beta_{j}^{i}(z)\right) \tilde{N}^{j}(d \tau, d z)\right\}
\end{gathered}
$$

Then, as $X_{t}^{t} \in \Pi$ by assumption, the lemma is proved.

We point out that this lemma does not need the matrix $\beta$ to be positive definite.

Proposition 3.2. Let $X_{s}^{t}(x)$ be the solution of problem (3.1). For all $p \geq 0$ and any terminal time $T>0$ there exists a constant $\mathrm{K}=\mathrm{K}(p, \mathrm{C}, T)$ such that for all $s, t \in[0, T]$ and $x, y \in\left(\mathbb{R}^{+}\right)^{\mathrm{N}}$ the following hold:

$$
\begin{aligned}
& \mathbb{E}\left[\left|X_{s}^{t}(x)\right|^{p}\right] \leq \mathrm{K}\left(1+|x|^{p}\right), \\
& \mathbb{E}\left[\left|X_{s}^{t}(x)-x\right|^{p}\right] \leq \mathrm{K}\left(1+|x|^{p}\right)(s-t)^{\frac{p}{2}}, \\
& \mathbb{E}\left[\sup _{0 \leq \tau \leq s}\left|X_{\tau}^{t}(x)-x\right|\right]^{p} \leq \mathrm{K}\left(1+|x|^{p}\right)(s-t)^{\frac{p}{2}}, \\
& \mathbb{E}\left[\left|X_{s}^{t}(x)-X_{s}^{t}(y)\right|^{p}\right] \leq \mathrm{K}|x-y|^{p} .
\end{aligned}
$$

Proof. These estimates follow as in [25, Lemma 3.1] and [13, Th.2.2, Th.2.3]. 
3.1. Existence and uniqueness of a solution for a BSDE. We introduce the following spaces (here $0 \leq t \leq T$ ):

$$
\begin{aligned}
\mathcal{S}^{2}= & \left\{Y_{t}: Y_{t} \text { is } \mathcal{F}_{t}-\right.\text { adapted, càdlàg, and } \\
& \left.\|Y\|_{\mathcal{S}^{2}}=\left\|\sup _{0 \leq t \leq T}\left|Y_{t}\right|\right\|_{L^{2}(\Omega)}<+\infty\right\} . \\
L^{2}(W)= & \left\{Z_{t}: Z_{t} \text { is } \mathcal{F}_{t}-\right.\text { progressively measurable and } \\
& \left.\|Z\|_{L^{2}(W)}=\left(\mathbb{E}\left[\int_{0}^{T}\left|Z_{t}\right|^{2} d t\right]\right)^{\frac{1}{2}}<+\infty\right\} . \\
L^{2}(\tilde{N})= & \left\{U: \Omega \times[0, T] \times E \rightarrow \mathbb{R}^{N}: U\right. \text { is measurable and } \\
& \left.\|U\|_{L^{2}(\tilde{N})}=\left(\mathbb{E}\left[\int_{0}^{T} \int_{E} U_{t}(z)^{2} \nu(d z) d t\right]\right)^{\frac{1}{2}}<+\infty\right\} . \\
\mathcal{B}^{2}= & \mathcal{S}^{2} \times L^{2}(W) \times L^{2}(\tilde{N}) .
\end{aligned}
$$

The following result is a combination of results in [32, Lemma 2.4], [24, Prop. 2.2] and [23].

Theorem 3.3. Let $G \in L^{2}\left(\Omega, \mathcal{F}_{T}, \mathcal{P}\right)$ and $f: \Omega \times[0, T] \times \mathbb{R}^{+} \times \mathbb{R}^{\mathrm{M}} \times L^{2}\left(E, \nu ; \mathbb{R}^{\mathrm{N}}\right) \rightarrow \mathbb{R}$ be progressively measurable with respect to all its variables and satisfy:

$$
\mathbb{E}\left[\int_{0}^{T}|f(t, 0,0,0)|^{2} d t\right]<+\infty
$$

and there exists $\mathrm{K}>0$ such that

$$
\left|f(t, y, \zeta, q)-f\left(t, y^{\prime}, \zeta^{\prime}, q^{\prime}\right)\right| \leq \mathrm{K}\left(\left|y-y^{\prime}\right|+\left|\zeta-\zeta^{\prime}\right|+|| u-u^{\prime} \|_{L^{2}(E)}\right),
$$

for all $0 \leq t \leq T$ and $y, y^{\prime} \in \mathbb{R}, \zeta, \zeta^{\prime} \in \mathbb{R}^{\mathrm{M}}, q, q^{\prime} \in L^{2}\left(E, \nu ; \mathbb{R}^{\mathrm{N}}\right)$. Then there exists a unique triple $(Y, Z, U) \in \mathcal{B}^{2}$ which solves the BSDE:

$$
Y_{t}=G+\int_{t}^{T} f\left(s, Y_{s}, Z_{s}, U_{s}\right) d s-\int_{t}^{T} Z_{s} d W_{s}-\int_{t}^{T} \int_{E} U_{s}(z) \tilde{N}(d s, d z),
$$

for $0 \leq t \leq T$.

We point out that the comparison result in [4] can be extended to the present case:

Proposition 3.4. Let us consider a progressively measurable function $h: \Omega \times[0, T] \times \mathbb{R} \times$ $\mathbb{R}^{\mathrm{M}} \times \mathbb{R}^{\mathrm{N}} \rightarrow \mathbb{R}$ satisfying

$$
\mathbb{E}\left[\int_{0}^{T}|h(\omega, t, 0,0,0)|^{2} d t\right]<+\infty,
$$

$h$ is globally Lipschitz continuous uniformly with respect to $(\omega, t) \in \Omega \times[0, T]$, and

$$
q \rightarrow h(t, y, \zeta, q) \text { is non decreasing for all }(t, y, \zeta) \in[0, T] \times \mathbb{R} \times \mathbb{R}^{\mathrm{M}} .
$$

Moreover, let $\gamma: \Omega \times[0, T] \times E \longrightarrow \mathbb{R}^{\mathrm{N} \times \mathrm{N}}$ be a measurable function satisfying assumption (A.4.i). Let us define

$$
f(\omega, t, y, \zeta, \phi)=h\left(\omega, t, y, \zeta, \int_{E} \phi(z) \gamma(\omega, t, z) \nu(d z)\right),
$$

for all $(\omega, t, y, \zeta, \phi) \in \Omega \times[0, T] \times \mathbb{R} \times \mathbb{R}^{\mathrm{M}} \times\left(L^{2}(E, \nu)\right)^{\mathrm{N}}$. Here we use the notation

$$
\int_{E} \phi(z) \gamma(\omega, t, z) \nu(d z)=\left(\int_{E} \phi_{1}(z) \gamma^{1}(\omega, t, z) \cdot \nu(d z), \ldots, \int_{E} \phi_{\mathrm{N}}(z) \gamma^{\mathrm{N}}(\omega, t, z) \cdot \nu(d z)\right) .
$$

With $G, G^{\prime} \in L^{2}\left(\Omega, \mathcal{F}_{T}, \mathcal{P}\right)$, let $(Y, Z, U)$ and $\left(Y^{\prime}, Z^{\prime}, U^{\prime}\right)$ be the unique solutions of (3.2) corresponding respectively to $G$ and $G^{\prime}$. If $G \leq G^{\prime}$ then

$$
Y_{t} \leq Y_{t}^{\prime} \text {, for all } 0 \leq t \leq T \text {. }
$$


This monotonicity result can be proved using Ito's formula, following the argument of the proof of the comparison principle for BSDEs without jumps, see [23].

In the following we analyze the case where both $G$ and, for each $t, y, \zeta$ and $q$, the process $\left\{f_{s}(y, \zeta, q), t \leq s \leq T\right\}$ are given functions of the stochastic process $X_{s}^{t}(x)$.

Let us suppose that

$$
g: \Pi \rightarrow \mathbb{R}, \quad f: \bar{\Pi}_{T} \times \mathbb{R} \times \mathbb{R}^{\mathrm{M}} \times \mathbb{R}^{\mathrm{N}} \rightarrow \mathbb{R}
$$

verify the following conditions:

(B.2) there exist a positive constant $\mathrm{C}$ and an integer $p$ such that, for all $(x, t) \in \bar{\Pi}_{T}$,

$$
|f(x, t, 0,0,0)| \leq \mathrm{C}\left(1+|x|^{p}\right) \quad|g(x)| \leq \mathrm{C}\left(1+|x|^{p}\right) .
$$

(B.3) $f=f(x, t, y, \zeta, q)$ is globally Lipschitz in $(y, \zeta, q)$, uniformly in $(x, t)$;

(B.4) for each $(x, t, y, \zeta) \in \bar{\Pi}_{T} \times \mathbb{R} \times \mathbb{R}^{\mathrm{M}}$ the function

$$
q \mapsto f(x, t, y, \zeta, q)
$$

is nondecreasing.

(B.5) the function $\gamma=\left(\gamma^{1}, \ldots, \gamma^{N}\right): \bar{\Pi}_{T} \times E \longrightarrow \mathbb{R}^{N}$ of Proposition 3.4 is such that there exists a positive constant $\mathrm{C}$

$$
\left|\gamma^{j}(x, t, z)-\gamma^{j}\left(x^{\prime}, t, z\right)\right| \leq \mathrm{C}\left|x-x^{\prime}\right|\left(1 \wedge|z|^{2}\right),
$$

for all $x, x^{\prime} \in \Pi, z \in E$ uniformly with respect to $t, j=1, \ldots, \mathrm{N}$.

Moreover, we introduce some additional assumptions that will be useful when proving the existence of a solution:

(B.6) there exists a modulus of continuity $\omega_{R}(\cdot)$ and a positive constant $\mathrm{C}_{R}$ such that, for all $t \in[0, T], x, x^{\prime} \in \Pi \cap B(0, R),|y| \leq R, \zeta \in \mathbb{R}^{\mathrm{M}}, q \in \mathbb{R}^{\mathrm{N}}, R>0$

$$
\begin{aligned}
& \left|f(x, t, y, \zeta, q)-f\left(x^{\prime}, t, y, \zeta, q\right)\right| \leq \omega_{R}\left(\left|x-x^{\prime}\right|(1+|\zeta|)\right), \\
& \left|g(x)-g\left(x^{\prime}\right)\right| \leq \mathrm{C}_{R}\left|x-x^{\prime}\right| .
\end{aligned}
$$

From now on we will use the notation $B^{+}(R)=\Pi \cap B(0, R)$, for $R>0$.

Let us consider the following BSDE:

$$
\begin{aligned}
Y_{s}^{t}(x) & =g\left(X_{T}^{t}(x)\right)+\int_{s}^{T} f\left(X_{\tau}^{t}(x), \tau, Y_{\tau}^{t}(x), Z_{\tau}^{t}(x), \int_{E} U_{\tau}^{t}(x, z) \gamma\left(X_{\tau}^{t}(x), \tau, z\right) \nu(d z)\right) d \tau \\
- & \int_{s}^{T} Z_{\tau}^{t}(x) d W_{\tau}-\int_{s}^{T} \int_{E} U_{\tau}^{t}(x, z) \tilde{N}(d \tau, d z), \quad t \leq s \leq T .
\end{aligned}
$$

Arguing as in [4, Corollary 2.3] and using Theorem 3.3, Lemma 3.1 and Proposition 3.2, the following theorem can be proved:

Theorem 3.5. For each $(x, t) \in \bar{\Pi}_{T}$ the BSDE (3.3) has a unique solution

$$
\left(Y^{t}(x), Z^{t}(x), U^{t}(x, \cdot)\right) \in \mathcal{B}^{2},
$$

and $(x, t) \mapsto Y_{t}^{t}(x)$ is a deterministic mapping from $\bar{\Pi}_{T}$ into $\mathbb{R}$.

Let us define

$$
u(x, t):=Y_{t}^{t}(x) .
$$

This deterministic function has the following properties:

Theorem 3.6. Under assumptions (B.1)-(B.5) the function $u: \bar{\Pi}_{T} \rightarrow \mathbb{R}$ is continuous. Moreover, there exist constants $\mathrm{C}$ and $p$ such that:

$$
|u(x, t)| \leq \mathrm{C}\left(1+|x|^{p}\right)(x, t) \in \bar{\Pi}_{T} .
$$

In addition, if $g$ and $f(\cdot, t, y, \zeta, q)$ are uniformly continuous, uniformly w.r.t. $(t, y, \zeta, q)$ and are bounded, then $u$ is uniformly continuous and bounded. 
Proof. We first establish the growth of $u$, and then that $u$ is Lipschitz continuous. As the solution $X_{s}^{t}(x)$ of $(3.1)$ remains inside $\Pi_{T}$, omitting the dependence on the starting point $x$, by repeating the arguments of [4, Prop. 2.2] we find that

$$
\|(Y, Z, U)\|_{\mathcal{B}^{2}}^{2} \leq c \mathbb{E}\left[\left|g\left(X_{s}^{t}\right)\right|^{2}+\int_{0}^{T}\left|f\left(X_{\tau}^{t}, \tau, Y_{\tau}^{t}, Z_{\tau}^{t}, \int_{E} U_{\tau}^{t}(\cdot, z) \gamma\left(X_{\tau}^{t}, \tau, z\right) \nu(d z)\right)\right|^{2} d s\right] .
$$

Using the assumptions (B.2) we obtain

$$
|u(x, t)| \leq\|(Y, Z, U)\|_{\mathcal{B}^{2}} \leq \mathrm{C}(T)\left(1+|x|^{p}\right)
$$

The proof of the Lipschitz continuity of $u$ follows as in [4, Prop. 2.5]. Define

$$
\hat{Y}_{s}^{t}(x)= \begin{cases}Y_{t}^{t}(x) & 0 \leq s \leq t \\ Y_{s}^{t}(x) & s>t\end{cases}
$$

It follows that

$$
\begin{aligned}
& \left|\hat{Y}_{t}^{t}(x)-\hat{Y}_{t^{\prime}}^{t^{\prime}}\left(x^{\prime}\right)\right|^{2}=\left|\hat{Y}_{0}^{t}(x)-\hat{Y}_{0}^{t^{\prime}}\left(x^{\prime}\right)\right|^{2} \leq\|(\hat{Y}, \hat{Z}, \hat{U})\|_{\mathcal{B}^{2}}^{2} \\
& \leq \mathrm{CE}\left[\left|g\left(X_{T}^{t}(x)\right)-g\left(X_{T}^{t^{\prime}}\left(x^{\prime}\right)\right)\right|^{2}\right. \\
& \quad+\int_{0}^{T} \mid \mathbf{1}_{[t, T]}(s) f\left(X_{s}^{t}(x), s, Y_{s}^{t}(x), Z_{s}^{t}(x), U_{s}^{t}(x, z)\right) \\
& \left.\quad-\mathbf{1}_{\left[t^{\prime}, T\right]} f\left(X_{s}^{t^{\prime}}\left(x^{\prime}\right), s, Y_{s}^{t^{\prime}}\left(x^{\prime}\right), U_{s}^{t^{\prime}}\left(x^{\prime}, z\right)\right) \mid d s\right] \\
& \leq \mathrm{K}\left(\left(1+|x|^{2}\right)^{2}\left(t-t^{\prime}\right)+\left|x-x^{\prime}\right|^{2}\right),
\end{aligned}
$$

where we have used assumptions (B). Moreover, if we assume that both $g$ and $f$ are uniformly continuous, then we can prove that $u$ is uniformly continuous.

3.2. Integro-Partial Differential Equation. Existence and Uniqueness. We now prove that the function $u(x, t)$ introduced in (3.4) provides a viscosity solution for an integropartial differential equation with terminal condition $g(x)$ and Hamiltonian

$$
\begin{aligned}
& H\left(x, t, u, \mathbf{x} D u, \mathbf{x} D^{2} u \mathbf{x}^{T}, \mathcal{I} u, \mathcal{J} u\right)=-\frac{1}{2} \operatorname{tr}\left[\sigma \sigma^{T} \mathbf{x} D^{2} u \mathbf{x}^{T}\right]-b \mathbf{x} D u \\
& -\sum_{j=1}^{\mathrm{N}} \mathcal{J}_{j} u-f\left(x, t, \mathbf{x} D u \sigma, \mathcal{I}_{1} u, \ldots, \mathcal{I}_{\mathrm{N}} u\right), \\
& \mathcal{J}_{j} u(x, t)=\int_{E}\left[u\left(x+\mathbf{x} \beta_{j}(x, z), t\right)-u(x, t)-\mathbf{x} \beta_{j}(x, z) D u(x, t)\right] \nu^{j}(d z), \\
& \mathcal{I}_{j} u(x, t)=\int_{E}\left[u\left(x+\mathbf{x} \beta_{j}(x, t, z), t\right)-u(x, t)\right] \gamma^{j}(x, t, z) \nu^{j}(d z),
\end{aligned}
$$

and the coefficients $b, \sigma, \beta$ and $\gamma$ satisfy all the assumptions in (A)-(B).

It will be shown that this problem can be considered as a particular case of the problem (1.1)-(1.2) where $u_{T}(x)=g(x)$. The techniques of Section 2 can easily be applied to this case even if the Hamiltonian depends explicitly on two integro operators, namely $\mathcal{J} u$ and $\mathcal{I} u$. This is not a complication, as it is explained in Remark 2.5 and in the introduction.

In the next theorem we show the link between (3.3)-(3.4) and (1.1) by proving that the unique solution of the stochastic problem is a viscosity solution of the integro-partial differential problem.

Theorem 3.7. The function $u(x, t)=Y_{t}^{t}(x),(x, t) \in \Pi_{T}$, is a viscosity solution of (1.1) with terminal condition (1.2). 
Proof. We have previously shown that $u(x, t)$ is deterministic, is continuous, and has polynomial growth. Moreover, it is easily seen that $u$ satisfies the terminal condition

$$
u(x, T)=Y_{T}^{T}(x)=u_{T}\left(X_{T}^{T}(x)\right)=u_{T}(x) .
$$

We will show that $u$ is a subsolution, the supersolution case being similar.

Let $\phi \in \mathcal{C}^{2}\left(\bar{\Pi}_{T}\right),(x, t)$ be a global maximum point for $u-\phi$ such that $u(x, t)-\phi(x, t)=0$. For simplicity, we suppose that $\phi$ has the same polynomial growth as $u$. It is easy to see that

$$
Y_{s}^{t}(x)=Y_{s}^{s}\left(X_{s}^{t}(x)\right)=u\left(X_{s}^{t}(x), s\right) .
$$

Choose now $h>0$ such that $t+h<T$. Omitting for simplicity the dependence on the starting point $x$, for $t \leq s \leq t+h$ we obtain

$$
\begin{aligned}
Y_{s}^{t}= & u\left(X_{t+h}^{t}, t+h\right)+\int_{s}^{t+h} f\left(X_{\tau}^{t}, \tau, Y_{\tau}^{t}, Z_{\tau}^{t}, \int_{E} U_{\tau}^{t}(x, z) \gamma\left(X_{\tau}^{t}, z\right) \nu(d z)\right) d \tau \\
& -\int_{s}^{t+h} Z_{\tau}^{t} d W_{\tau}-\int_{s}^{t+h} \int_{E} U_{\tau}^{t}(x, z) \tilde{N}(d t, d z) .
\end{aligned}
$$

Still omitting the dependence on $x$, consider now the following process

$$
\begin{aligned}
\bar{Y}_{s}^{t}= & \phi\left(X_{t+h}^{t}(x), t+h\right)+\int_{s}^{t+h} f\left(X_{\tau}^{t}, \tau, \bar{Y}_{\tau}^{t}, \bar{Z}_{\tau}^{t}, \int_{E} \bar{U}_{\tau}^{t}(x, z) \gamma\left(X_{\tau}^{t}, z\right) \nu(d z)\right) d \tau \\
& -\int_{s}^{t+h} \bar{Z}_{\tau}^{t} d W_{\tau}-\int_{s}^{t+h} \int_{E} \bar{U}_{\tau}^{t}(x, z) \tilde{N}(d t, d z) .
\end{aligned}
$$

As $(x, t)$ is a global maximum point, from Proposition 3.4, comparison holds

$$
\bar{Y}_{s}^{t}(x) \geq Y_{s}^{t}(x), t \leq s \leq t+h .
$$

In particular, for $s=t$ there holds

$$
\bar{Y}_{t}^{t}(x) \geq u(x, t)=\phi(x, t) .
$$

Let us now define

$$
\begin{aligned}
\psi(y, s)= & \partial_{s} \phi(y, s)+\frac{1}{2} \operatorname{tr}\left[\sigma(y, t) \sigma(y, t)^{T} \mathbf{y} D^{2} \phi(y, s) \mathbf{y}^{T}\right]+b(y, s) \mathbf{y} D \phi(y, s) \\
& +\sum_{j=1}^{\mathrm{N}} \mathcal{J}_{j} \phi(y, s), \\
\Phi_{j}(y, s, z)= & \phi\left(y+\beta_{j}(y, s, z), s\right)-\phi(y, s),
\end{aligned}
$$

with $\Phi(y, s, z)=\left(\Phi_{1}(y, s, z), \ldots, \Phi_{\mathrm{N}}(y, s, z)\right) \in \mathbb{R}^{\mathrm{N}}$. By the generalized Ito's formula,

$$
\begin{aligned}
& \phi\left(X_{s}^{t}(x), s\right)=\phi\left(X_{t+h}^{t}(x), t+h\right)-\int_{s}^{t+h} \psi\left(X_{\tau}^{t}(x), \tau\right) d \tau \\
& \quad-\int_{s}^{t+h} \mathbf{X}_{\tau}^{t}(x) \cdot D \phi\left(X_{\tau}^{t}(x), \tau\right) \sigma\left(X_{\tau}^{t}(x), \tau\right) d W_{\tau}-\int_{s}^{t+h} \int_{E} \Phi\left(\tau, X_{\tau}^{t}(x)\right) \tilde{N}(d t, d z) .
\end{aligned}
$$

Let us now define the new processes

$$
\begin{aligned}
\hat{Y}_{s}^{t}(x) & :=\bar{Y}_{s}^{t}-\phi\left(X_{s}^{t}(x), s\right) \\
\hat{Z}_{s}^{t}(x) & :=\bar{Z}_{s}^{t}(x)-\mathbf{X}_{s}^{t} D \phi\left(X_{s}^{t}(x), s\right) \sigma\left(X_{s}^{t}(x), s\right), \\
\hat{U}_{s}^{t}(x, z) & :=\bar{U}_{s}^{t}(x, z)-\Phi\left(X_{s}^{t}(x), s, z\right)
\end{aligned}
$$


Then the triplet $(\hat{Y}, \hat{Z}, \hat{U})$ is the solution of the following BSDE:

$$
\begin{aligned}
\hat{Y}_{s}^{t}(x)=\int_{s}^{t+h} & {\left[f\left(X_{\tau}^{t}(x), \tau, \bar{Y}_{\tau}^{t}(x), \bar{Z}_{\tau}^{t}(x), \int_{E} \bar{U}_{\tau}^{t}(x, z) \gamma\left(X_{\tau}^{t}(x), z\right) \nu(d z)\right)\right.} \\
+ & \left.\psi\left(X_{\tau}^{t}(x), \tau\right)\right] d \tau-\int_{s}^{t+h} \hat{Z}_{\tau}^{t}(x) d W_{\tau}-\int_{s}^{t+h} \int_{E} \hat{U}_{\tau}^{t}(x) \tilde{N}(d t, d z) .
\end{aligned}
$$

Using standard techniques it is easy to prove that

$$
\begin{aligned}
& \mathbb{E}\left[\left|\hat{Y}_{s}^{t}(x)\right|^{2}\right]+\mathbb{E}\left[\int_{s}^{t+h}\left|\hat{Z}_{\tau}^{t}(x)\right|^{2} d \tau\right]+\mathbb{E}\left[\int_{s}^{t+h} \int_{E}\left|\hat{U}_{\tau}^{t}(x, z)\right|^{2} \nu(d z) d \tau\right] \\
& \leq 2 \mathbb{E}\left[\int_{s}^{t+h}\left|\hat{Y}_{\tau}^{t}(x)\right| \mid f\left(X_{\tau}^{t}(x), \tau, \bar{Y}_{\tau}^{t}(x), \bar{Z}_{\tau}^{t}(x), \int_{E} \bar{U}_{\tau}^{t}(x, z) \gamma\left(X_{\tau}^{t}(x), z\right) \nu(d z)\right)\right. \\
& \left.\quad+\psi\left(X_{\tau}^{t}(x), \tau\right) \mid d \tau\right] \\
& \leq 2 \mathrm{KE}\left[\int_{s}^{t+h}\left|\hat{Y}_{\tau}^{t}(x)\right|\left(1+\left|\hat{Y}_{\tau}^{t}(x)\right|+\left|\hat{Z}_{\tau}^{t}(x)\right|+|| \hat{U}_{z}^{t}(x) \|_{L^{2}(E)}\right) d \tau\right]
\end{aligned}
$$

therefore we obtain

$$
\begin{aligned}
& \mathbb{E}\left[\left|\hat{Y}_{s}^{t}(x)\right|^{2}\right]+\frac{1}{2}\left\{\mathbb{E}\left[\int_{s}^{t+h}\left|\hat{Z}_{\tau}^{t}(x)\right|^{2} d \tau\right]+\mathbb{E}\left[\int_{s}^{t+h} \int_{E}\left|\hat{U}_{\tau}^{t}(x, z)\right|^{2} \nu(d z) d \tau\right]\right\} \\
& \leq 2 \mathrm{~K} \mathbb{E}\left[\int_{s}^{t+h}\left(\left|\hat{Y}_{\tau}^{t}\right|+\left|\hat{Y}_{\tau}^{t}\right|^{2}\right) d \tau\right],
\end{aligned}
$$

where we have used (B.3) for $f$ and the polynomial growth of $\phi$. Using now assumptions (B) and Proposition 3.2 we obtain

$$
\mathbb{E}\left[\left|\hat{Y}_{s}^{t}\right|^{2}\right] \leq 4 \mathrm{~K}\left(h+\mathbb{E}\left[\int_{s}^{t+h}\left|\hat{Y}_{\tau}^{t}\right|^{2} d \tau\right]\right) .
$$

By Gronwall's lemma,

$$
\mathbb{E}\left[\left|\hat{Y}_{\tau}^{t}\right|^{2}\right] \leq \mathrm{K} h
$$

for some $\mathrm{K}>0$. In the same way, if $0<h<1$, we obtain

$$
\frac{1}{h}\left(\mathbb{E}\left[\int_{s}^{t+h}\left|\hat{Z}_{\tau}^{t}\right|^{2} d \tau\right]+\mathbb{E}\left[\int_{s}^{t+h} \int_{E}\left|\hat{U}_{\tau}^{t}(z)\right|^{2} \nu(z) d \tau\right]\right) \leq \mathrm{K} \sqrt{h} .
$$

Let us suppose now that it holds

$$
\partial_{t} \phi(x, t)-H\left(x, t, u(x, t), \mathbf{x} D \phi(x, t), \mathbf{x} D^{2} \phi(x, t) \mathbf{x}^{T}, \mathcal{J} \phi(x, t), \mathcal{I} \phi(x, t)\right)<0 .
$$

Omitting the dependence of $\psi$ and $\phi$ on $\left(X_{\tau}^{t}(x), \tau\right)$, let us define

$$
\xi_{h}:=\frac{1}{h} \mathbb{E}\left[\int_{t}^{t+h}\left[\psi+f\left(X_{\tau}^{t}(x), \tau, \phi, D \phi \sigma, \mathcal{I} \phi\right)\right] d \tau\right] .
$$

From the previous hypotheses there exist $\delta>0$ and $\bar{h}>0$ such that for all $0<h \leq \bar{h}$ :

$$
\xi_{h} \leq-\delta<0 .
$$

Remembering the definition of $\hat{Y}_{s}^{t}(x)$, that $(x, t)$ is a global maximum point for $u-\phi$ and the monotonicity of the solution of (3.2) with respect the initial data, we have

$$
\hat{Y}_{t}^{t}(x)=\bar{Y}_{t}^{t}(x)-\phi(x, t) \geq u(x, t)-\phi(x, t)=0 .
$$

Therefore, omitting the dependence on the starting point $x$,

$$
0 \leq \frac{1}{h} \hat{Y}_{t}^{t}=\frac{1}{h} \mathbb{E}\left[\int_{t}^{t+h}\left[\psi\left(X_{\tau}^{t}, \tau\right)+f\left(X_{\tau}^{t}, \tau, \bar{Y}_{\tau}^{t}, \bar{Z}_{\tau}^{t}, \int_{E} \bar{U}_{\tau}^{t} \gamma\left(X_{\tau}^{t}, z\right) \nu(d z)\right)\right] d \tau\right] .
$$


Then, still omitting the dependence on $x$, for $0<h \leq \bar{h}$ we have

$$
\begin{aligned}
\delta \leq & \frac{1}{h} \hat{Y}_{t}^{t}-\xi_{h}=\frac{1}{h} \mathbb{E}\left[\int _ { t } ^ { t + h } \left[f\left(X_{\tau}^{t}, \tau, \bar{Y}_{\tau}^{t}, \bar{Z}_{\tau}^{t}, \int_{E} \bar{U}_{\tau}^{t} \gamma\left(X_{\tau}^{t}, z\right) \nu(d z)\right)\right.\right. \\
& \left.\left.-f\left(X_{\tau}^{t}, \tau, \phi\left(X_{\tau}^{t}, \tau\right),(D \phi \sigma)\left(X_{\tau}^{t}, \tau\right), \mathcal{I} \phi\left(X_{\tau}^{t}, \tau\right)\right)\right] d \tau\right] \\
\leq & \frac{\mathrm{K}}{h} \mathbb{E}\left[\int_{t}^{t+h}\left(\left|\hat{Y}_{\tau}^{t}\right|+\left|\hat{Z}_{\tau}^{t}\right|+\left\|\hat{U}_{\tau}^{t}\right\|_{L^{2}(E)}\right) d \tau\right] \leq \mathrm{K}\left[c_{1} \sqrt{h}+c_{2} \sqrt[4]{h}\right] \leq \mathrm{C} \sqrt[4]{h},
\end{aligned}
$$

which is a contradiction. Hence we must have

$$
-\partial_{t} \phi(x, t)+H\left(x, t, u(x, t), \mathbf{x} D \phi(x, t), \mathbf{x} D^{2} \phi(x, t) \mathbf{x}^{T}, \mathcal{J} \phi(x, t), \mathcal{I} \phi(x, t)\right) \leq 0,
$$

which means that $u$ is a subsolution.

Using the result of Section 2 we also have the uniqueness of the solution of the integropartial differential problem.

Theorem 3.8. Let us suppose that assumptions $(\mathbf{B})$ hold. Then the function $u(x, t)$ defined in (3.4) is the unique viscosity solution of (1.1)-(1.2), with Hamiltonian (3.5) and terminal data $g(x)$. The viscosity solution $u$ has the same polynomial growth as the function $f$ and the terminal data $g(x)$.

Proof. By Corollary 2.11 it suffices to verify the assumptions (A.1)-(A.3) of Section 2 for the Hamiltonian (3.5). As a consequence of the presence of the integral term $\mathcal{I} u$, we also have to take into account assumption (A.4), as explained in Remark 2.5. This does not introduce new difficulties and follows straightforward from (B). This proves the result.

\section{The Pricing Problem}

In this section we apply the results from Sections 2 and 3 to the pricing of European and American derivatives in Lévy markets. To this end, let us consider $\mathrm{N}$ Lévy processes with the following dynamics

$$
\begin{aligned}
d L_{t}^{i}= & \mu^{i}(t) d t+\sum_{j=1}^{\mathrm{M}} \sigma_{j}^{i}(t) d W_{t}^{j}+\sum_{j=1}^{\mathrm{N}} \int_{|z|<1} \eta_{j}^{i}(t) z^{j} \tilde{N}^{j}(d t, d z) \\
& +\sum_{j=1}^{\mathrm{N}} \int_{|z| \geq 1} \eta_{j}^{i}(t) z^{j} N^{j}(d t, d z), \quad i=1, \ldots, \mathrm{N},
\end{aligned}
$$

where $\sigma(t) \in \mathbb{R}^{\mathrm{N} \times \mathrm{M}}$ matrix, $1 \leq \mathrm{M} \leq \mathrm{N}$, and $\sigma(t) \sigma^{T}(t)$ possibly degenerate, $\eta(t) \in \mathbb{R}^{\mathrm{N} \times \mathrm{N}} ; \mu$, $\sigma$ and $\eta$ are assumed to be deterministic functions. Consider a financial market where the risk-free asset, $B_{t}$, and the risky assets, $X_{t}=\left(X_{t}^{1}, \ldots, X_{t}^{N}\right)$, evolve according to

$$
\left\{\begin{array}{l}
B_{t}=e^{r t} \\
X_{t}^{i}=e^{L_{t}^{i}}, \quad i=1, \ldots, \mathrm{N},
\end{array}\right.
$$

where $r>0$ and $t>0$. By the generalized Ito's formula, the $X_{t}$ dynamics are given by (3.1) with parameters $b\left(X_{t}, t\right) \in \mathbb{R}^{\mathrm{N}}, \sigma\left(X_{t}, t\right) \in \mathbb{R}^{\mathrm{N} \times \mathrm{M}}, \beta\left(X_{t}, t, z\right) \in \mathbb{R}^{\mathrm{N} \times \mathrm{N}}$ taking the following form

$$
\begin{array}{ll}
b^{i}\left(X_{t}\right) & =\mu^{i}+\frac{1}{2} \sum_{j=1}^{\mathrm{M}}\left(\sigma_{j}^{i}\right)^{2}+\sum_{j=1}^{\mathrm{N}} \int_{E}\left(e^{\eta_{j}^{i} z^{j}}-1-\eta_{j}^{i} z^{j} \mathbf{1}_{|z|<1}\right) \nu^{j}(d z), \\
\sigma\left(X_{t}\right) & =\sigma \\
\beta_{j}^{i}\left(X_{t}, z\right) & =e^{\eta_{j}^{i} z^{j}}-1,
\end{array}
$$

where, for simplicity, we have omitted the $t$ dependence. From these equalities it comes out that $b$ and $\beta$ are deterministic functions. 
In the following we shall assume that the coefficients $b, \sigma$ verify assumption (B.1), $\beta$ and the measures $\nu=\left(\nu^{1}, \ldots, \nu^{\mathrm{N}}\right)$ verify assumptions (A.2) and (A.1) of Section 2 respectively, and that the interest rate $r$ is bounded from below.

The presence of the jump components in the price dynamics makes the market incomplete. Here we assume to be in an equivalent martingale setting. This assumption can be made without loss of generality, as shown by the following result:

Proposition 4.1. [26, Prop. 2.19] Let $X$ be a Lévy process with Lévy-Kintchine triplet $\left(\mu, \sigma \sigma^{T}, \nu\right)$ under some probability measure $\mathcal{P}$. Then the following two conditions are equivalent:

(a) There is a probability measure $\mathcal{Q} \stackrel{\text { loc }}{\sim} \mathcal{P}$ such that $X$ is a $\mathcal{Q}$-Lévy process with triplet $\left(\mu^{\prime}, \sigma^{\prime}\left(\sigma^{\prime}\right)^{T}, \nu^{\prime}\right)$.

(b) The following conditions hold:

(i) $\nu^{\prime}(d z)=k(z) \nu(d z)$ for some Borel function $k: \mathbb{R}^{N} \longrightarrow \Pi$.

(ii) $\left(\mu^{\prime}\right)^{i}=\mu^{i}+\sum_{j=1}^{\mathrm{N}} \int_{E} \eta_{j}^{i} z^{i}\left(k^{j}(z)-1\right)\left(\nu^{\prime}\right)^{j}(d z)+\sum_{j=1}^{\mathrm{M}} \sigma_{j}^{i} \alpha^{j}$ for some $\alpha \in \mathbb{R}^{\mathrm{M}}$.

(iii) $\sigma^{\prime}=\sigma$.

(iv) $\int_{E}\left(1-\sqrt{k^{i}(z)}\right)^{2} \nu^{i}(d z)<\infty$, for all $i=1, \ldots, \mathrm{N}$.

We conclude that if a change of measure is needed, the $X_{t}$ dynamics with respect to the new measure are still given by (3.1), modulo a change in the drift coefficient:

$\left(b^{\mathcal{Q}}\right)^{i}\left(X_{t}, t\right)=\mu^{i}+\frac{1}{2} \sum_{j=1}^{\mathrm{M}}\left(\sigma_{j}^{i}\right)^{2}(t)+\sum_{j=1}^{\mathrm{N}} \int_{E}\left[e^{\eta_{j}^{i}(t) z^{j}}-1-\eta_{j}^{i}(t) z^{j} \mathbf{1}_{|z|<1}\right] k^{j}(z) \nu^{j}(d z)+\sum_{j=1}^{\mathrm{M}} \sigma_{j}^{i} \alpha^{j}$,

and with a new compensated martingale measure $\tilde{N}^{\mathcal{Q}}$. We point out that the coefficient $b^{\mathcal{Q}}$ still satisfies assumption (B.1).

In an equivalent martingale setting, the discounted prices of the assets are martingales, and therefore the parameters have to satisfy

$$
b^{i}\left(X_{t}, t\right)-r=0, \text { for all } i=1, \ldots, \mathrm{N} .
$$

4.1. The European Derivatives. Consider the price $Y_{t}$ of a European derivative constructed on the assets $X_{t}$ with maturity $T$ and payoff $u_{T}\left(X_{T}\right)$. The discounted price of the derivative, $\hat{Y}_{t}=e^{-r t} Y_{t}$, is a martingale, and therefore the price $Y_{t}$ is given as

$$
Y_{t}=\mathbb{E}\left[e^{-t(T-t)} u_{T}\left(X_{T}\right) \mid \mathcal{F}_{t}\right]
$$

using Ito's calculus, the dynamic of $Y_{t}$ is given as

$$
d Y_{t}=r Y_{t} d t+e^{r t} d \hat{Y}_{t} .
$$

Using the martingale representation theorem, there exist two processes $\hat{Z}_{t} \in L^{2}(W)$ and $\hat{U}_{t} \in L^{2}(\tilde{N})$ such that

$$
\hat{Y}_{t}=\hat{Y}_{0}+\int_{0}^{t} \hat{Z}_{s} d W_{s}+\int_{0}^{t} \int_{E} \hat{U}_{s}(z) \tilde{N}(d t, d z)
$$

(see [18, Chap. 2, Thms. 6.6-6.7] and [32, Lemma 2.3]) therefore there exists a triple $\left(Y_{t}, Z_{t}, U_{t}\right)$ satisfying the $\operatorname{BSDE}(3.2)$ where

$$
f\left(Y_{t}, Z_{t}, U_{t}\right)=r Y_{t} .
$$

Let us now assume that there exists a deterministic function $u$ giving the price of the derivative. Then we have the following result. 
Proposition 4.2. The price $u$ of a European derivative with maturity $T$ and payoff $u_{T}$ is a viscosity solution of the following equation

$$
-\partial_{t} u+H\left(x, t, u, \mathbf{x} D u, \mathbf{x} D^{2} u \mathbf{x}^{T}, \mathcal{J} u\right)=0,
$$

with terminal condition $u_{T}(x), x \in \Pi$. Here

$$
H\left(x, t, u, \mathbf{x} D u, \mathbf{x} D^{2} u \mathbf{x}^{T}, \mathcal{J} u\right)=-\frac{1}{2} \operatorname{tr}\left[\sigma(t) \sigma(t)^{T} \mathbf{x} D^{2} u \mathbf{x}^{T}\right]-b \mathbf{x} D u+r u-\sum_{j=1}^{\mathrm{N}} \mathcal{J}_{j} u
$$

and

$$
\mathcal{J}_{j} u(x, t)=\int_{E}\left[u\left(\mathbf{x} e^{(\eta(t) z)_{j}}, t\right)-u(x, t)-\mathbf{x}\left(e^{\eta(t) z}-\mathbf{1}_{\mathrm{N}}\right) D u\right] \nu^{j}(d z),
$$

where $\mathbf{1}_{\mathrm{N}}$ is the $\mathrm{N}$-dimensional vector $(1, \ldots, 1)$.

Proof. This result is a consequence of the theory presented in Section 3, since we have the following identifications:

$$
\begin{array}{ll}
Y_{s}^{t}(x) & =e^{-r(s-t)} u\left(X_{s}^{t}(x), t\right), \\
f\left(X_{t}, t, Y_{t}, Z_{t}, U_{t}\right) & =r Y_{t} .
\end{array}
$$

Arguing as in section 3, we can now state the following result.

Theorem 4.3. There exists a unique viscosity solution $u(x, t)$ of (4.3) with terminal data $u(x, T)=u_{T}(x)$. Moreover, $u(x, t)$ is given as the solution of the BSDE (3.2) with $f$ as in $(4.2)$.

Proof. We have just shown that the pricing problem can be formulated as a special case of the more general problem described in Section 3. Hence to prove that a solution in the viscosity sense exists and is unique, we need only to check that it satisfies the assumptions of Sections 2 and 3. Moreover, the present integro-partial differential equation does not depend on the integro operator $\mathcal{I} u$, and therefore its study reduces exactly to Corollary 2.11 and Theorem 3.7. Assumptions (B) are easily checked as $r$ is supposed to be bounded from below. To prove uniqueness it suffices to verify that the Hamiltonian $H$ satisfies assumption (A.3). Assumptions (A.1.i) - (A.1.iii) are consequences of $(\mathbf{B})$, of the boundedness of $r$ and of the linearity of the Hamiltonian $H$ with respect to its arguments.

To check assumption (A.3.iv), suppose $x, y \in \mathcal{Q}(r), t \in[0, T], u \in[-R, R], \mathcal{J} \in \mathbb{R}$, and $M, N \in \mathcal{S}^{\mathrm{N}}$ satisfy

$$
\left(\begin{array}{cc}
M & 0 \\
0 & -N
\end{array}\right) \leq \frac{3}{\varepsilon}\left(\begin{array}{cc}
I & -I \\
-I & I
\end{array}\right)
$$

Then

$$
\begin{aligned}
& H\left(x, t, u, \frac{1}{\varepsilon} \mathbf{x}(x-y), \mathbf{x} M \mathbf{x}^{T}, \mathcal{J}\right)-H\left(y, t, u, \frac{1}{\varepsilon} \mathbf{y}(x-y), \mathbf{y} N \mathbf{y}^{T}, \mathcal{J}\right) \\
& \geq-\frac{3}{2 \varepsilon} \operatorname{tr}\left(\sigma \sigma^{T}\right)|\mathbf{x}-\mathbf{y}|^{2}-\frac{1}{\varepsilon}(x-y)(\mathbf{x}-\mathbf{y})\left(\mu+\sigma \sigma^{T}\right),
\end{aligned}
$$

and the calculations are carried out as in [11, Example 3.6].

4.2. The American Derivatives. Let us suppose we are in a market described by (4.1) and that we are equipped with an equivalent martingale measure.

Let $\mathcal{T}_{t, T}$ denote the set of all stopping times between $t$ and $T$. Let $Y_{t}$ be the price of the American derivative with maturity $T$ and exercise price $u_{T}$. Then $Y_{t}$ is given as

$$
Y_{t}=\sup _{\tau \in \mathcal{T}_{t}, T} \mathbb{E}\left[e^{-r(\tau-t)} u_{T}\left(X_{\tau}\right) \mid X_{t}=x\right]=\sup _{\tau \in \mathcal{T}_{t, T}} \mathbb{E}\left[e^{-r(\tau-t)} u_{T}\left(X_{\tau}^{t}(x)\right)\right] .
$$


For the general theory of American derivatives and the related control problem we refer to $[12,17,19]$. The related integro-partial differential equation is

$$
\min \left(-\partial_{t} u+H\left(x, t, u, \mathbf{x} D u, \mathbf{x} D^{2} u \mathbf{x}^{T}, \mathcal{J} u\right), u-u_{T}\right)=0,
$$

for $(x, t) \in \Pi_{T}$, with terminal data

$$
u(x, T)=u_{T}(x),
$$

for $x \in \Pi$. We intend to show that the function $u$ defined in (3.2) is a viscosity solution of the integro-partial differential equation (4.5).

We recall here a definition of viscosity solutions that is suitable for the obstacle problem (4.5)-(4.6).

Definition 4.1. An upper semicontinuous function $u: \Pi_{T} \rightarrow \mathbb{R}$ is a viscosity subsolution of equation (4.5) if

$$
-\partial_{t} u+H\left(x, t, u, \mathbf{x} D u, \mathbf{x} D^{2} u \mathbf{x}^{T}, \mathcal{J} u\right) \leq 0,
$$

in the viscosity sense for all $(x, t) \in \Pi_{T}$ such that $u(x, t)>u_{T}(x)$.

A lower semicontinuous function $u: \Pi_{T} \rightarrow \mathbb{R}$ is a viscosity supersolution of equation (4.5) if

$$
-\partial_{t} u+H\left(x, t, u, \mathbf{x} D u, \mathbf{x} D^{2} u \mathbf{x}^{T}, \mathcal{J} u\right) \geq 0,
$$

in the viscosity sense for all $(x, t) \in \Pi_{T}$ such that $u(x, t) \geq u_{T}(x)$ and the parabolic subjet $\mathcal{P}^{-}=\emptyset$ for all $(x, t) \in\left(\mathbb{R}^{+}\right)^{\mathrm{N}} \times[0, T]$ such that $u(x, t)<u_{T}(x)$.

If, in addition,

$$
\left.u(x, T) \leq u_{T}(x) \text { (respectively, } \geq\right),
$$

for all $x \in \Pi$, then $u$ is a viscosity subsolution (respectively, viscosity supersolution) of the obstacle problem (4.5)-(4.6).

An arbitrary function $u: \Pi_{T} \rightarrow \mathbb{R}$ is a viscosity solution for the obstacle problem (4.5)(4.6) if the upper and lower semicontinuous envelopes of $u$ are viscosity sub- and supersolutions, respectively.

Because of Lemma 3.1, the Dynamic Programming Principle (DPP) established in [25, Prop. 3.1 and Prop. 3.2] holds for our problem (4.5)-(4.6). Hence the following results can be proved along the lines of [25, Prop. 3.3] and [25, Th. 3.1].

Theorem 4.4. There exists a deterministic function $u \in \mathcal{C}\left(\Pi_{T}\right)$ such that

$$
u\left(X_{t}, t\right)=Y_{t},
$$

where $Y_{t}$ is given as in (4.4). Moreover under assumptions (B) of Section 3, u satisfies the conclusions of Theorem 3.6. In addiction, there exists a positive constant $\mathrm{C}_{R}$ such that for all $t, s \in[0, T]$, for all $x, y \in \mathcal{Q}(R)$

$$
|u(x, t)-u(y, s)| \leq \mathrm{C}_{R}\left(|t-s|^{\frac{1}{2}}+|x-y|\right) .
$$

Proof. By definition, the discounted price of the American derivative, $\hat{Y}_{t}=e^{-r t} Y_{t}$, is a martingale. Therefore, because of the martingale representation theorem, there exists a pair $\left(\hat{Z}_{t}, \hat{U}_{t}\right) \in L^{2}(W) \times L^{2}(\tilde{N})$ such that $\hat{Y}_{t}$ solves a BSDE of the kind of $(3.2)$, the same holding for $Y_{t}$ for a suitable pair $\left(Z_{t}, U_{t}\right)$. From the results in Section 3 (Theorem 3.5) we can define a deterministic function $u$ as in (3.4), satisfying a polynomial rate of growth as in Theorem 3.6. If $u_{T}$ is continuous, arguing as in [25, Prop. 3.3], using the Dynamic Programming Principle, we get that $u$ is continuous and verifies

$$
|u(x, t)-u(y, s)| \leq \mathrm{C}_{R}\left(|t-s|^{\frac{1}{2}}+|x-y|\right) .
$$

Theorem 4.5. Under assumptions (B) of Section 3 the function $u$ in (4.4) is a viscosity solution of (4.5)-(4.6). 
Proof. We divide the proof in two steps, proving at first that $u$ is a supersolution and afterwards that it is a subsolution.

Let $\phi \in \mathcal{C}^{2}\left(\bar{\Pi}_{T}\right) \cap \bigcup_{n<n_{o}} L^{\infty}\left(0, T ; \mathcal{P}_{n}\left(\Pi_{T}\right)\right)$ and suppose that $(x, t) \in \Pi_{T}$ is a global minimum point of $u-\phi$ such that $(u-\phi)(x, t)=0$. By definition, for $t<h<T$

$$
u(x, t) \geq \mathbb{E}\left[e^{-r(h-t)} u\left(X_{h}^{t}(x), h\right)\right] .
$$

Subtracting $\phi(x, t)$ and using the hypotheses, we get

$$
0 \geq \mathbb{E}\left[e^{-r(h-t)} \phi\left(X_{h}^{t}(x), h\right)-\phi(x, t)\right] .
$$

Applying Ito's formula to $e^{-r t} \phi\left(X_{t}, t\right)$ we obtain

$$
\frac{1}{h-t} \mathbb{E}\left[\int_{t}^{h}\left(\partial_{t} \phi-H\left(X_{s}^{t}(x), s, \phi, \mathbf{X}_{s}^{t} D \phi, \mathbf{X}_{s}^{t} D^{2} \phi \mathbf{X}_{s}^{t^{T}}, \mathcal{J} \phi\right)\right) d s\right] \leq \omega(h-t),
$$

where $\omega$ is a modulus of continuity and the function $\phi$ is evaluated at $\left(X_{s}^{t}(x), s\right)$. Sending now $h$ to $t$ we obtain

$$
-\partial_{t} \phi(x, t)+H\left(x, t, u(x, t), \mathbf{x} D u(x, t), \mathbf{x} D^{2} u(x, t) \mathbf{x}^{T}, \mathcal{J} u(x, t)\right) \geq 0 .
$$

On the other hand, by definition of American option we have

$$
u(x, t) \geq u_{T}(x),
$$

and therefore we have proved the viscosity supersolution inequality.

To prove that $u$ is a subsolution we will use the equivalent definition of $u$ by the DPP. Let $\phi \in \mathcal{C}^{2}\left(\bar{\Pi}_{T}\right) \cap \bigcup_{n<n_{\circ}} L^{\infty}\left(0, T ; \mathcal{P}_{n}\left(\Pi_{T}\right)\right)$ and suppose that $(x, t) \in \Pi_{T}$ is a global maximum point of $u-\phi$ such that $(u-\phi)(x, t)=0$. By definition we already know that $u(x, t) \geq u_{T}(x)$.

Let us suppose that the equality is met, then the subsolution inequality is obviously satisfied and we are done. On the contrary, suppose that the strict inequality holds and define

$$
\varepsilon=\frac{u(x, t)-u_{T}(x)}{2}>0
$$

and the related stopping time

$$
\tau_{x, t}^{\varepsilon}=\inf \left\{t \leq s \leq T, u\left(X_{s}^{t}(x), s\right) \leq u_{T}\left(X_{s}^{t}(x)\right)+\varepsilon\right\} .
$$

Using [25, Prop. 3.2], for all $h>t$ we have

$$
\begin{aligned}
u(x, t)= & \mathbb{E}\left[e^{-r\left(h \wedge \tau_{x, t}^{\varepsilon}-t\right)} u\left(X_{h \wedge \tau_{x, t}^{\varepsilon}}^{t}(x), h \wedge \tau_{x, t}^{\varepsilon}\right)\right. \\
& \left.+\int_{t}^{h \wedge \tau_{x, t}^{\varepsilon}} e^{-r(s-t)} f\left(X_{s}^{t}(x), s, u, \mathbf{X}_{s}^{t} D u\right) d s\right]
\end{aligned}
$$

where the function $u$ in the integral part is evaluated in $\left(X_{s}^{t}(x), s\right)$. Subtracting $\phi(x, t)$, as $(x, t)$ is a maximum point, we get

$$
\begin{aligned}
0 \leq & \frac{1}{h-t} \mathbb{E}\left[e^{-r\left(h \wedge \tau_{\boldsymbol{x}, t}^{\varepsilon}-t\right)} \phi\left(X_{h \wedge \tau_{\boldsymbol{x}, t}^{\varepsilon}}^{t}(x), h \wedge \tau_{x, t}^{\varepsilon}\right)-\phi(x, t)\right. \\
& \left.+\int_{t}^{h \wedge \tau_{\boldsymbol{x}, t}^{\varepsilon}} e^{-r(s-t)} f\left(X_{s}^{t}(x), s, u\left(X_{s}^{t}(x), s\right), \mathbf{X}_{s}^{t}(x) D u\left(X_{s}^{t}(x), s\right)\right) d s\right] .
\end{aligned}
$$


As before, using Ito's formula on $e^{-r t} \phi\left(X_{t}, t\right)$ and properties of the process $X_{t}$ we obtain

$$
\begin{aligned}
\omega(h-t) \leq & \frac{1}{h-t} \mathbb{E}\left[\int_{t}^{h \wedge \tau_{x, t}^{\varepsilon}}\left(\partial_{t} \phi-H\left(X_{s}^{t}(x), s, \phi, \mathbf{X}_{s}^{t} D \phi, \mathbf{X}_{s}^{t} D^{2} \phi \mathbf{X}_{s}^{t^{T}}, \mathcal{J} \phi\right)\right) d s\right] \\
\leq & \sup _{t \leq s \leq h \wedge \tau_{x, t}^{\varepsilon}}\left(\partial_{t} \phi(x, s)-H\left(x, s, \phi(x, s), \mathbf{x} D \phi, \mathbf{x} D^{2} \phi \mathbf{x}^{T}, \mathcal{J} \phi(x, s)\right)\right) \\
& \quad \times \mathbb{E}\left[\frac{h \wedge \tau_{x, t}^{\varepsilon}-t}{h-t}\right],
\end{aligned}
$$

where the function $\phi$ on the first line is evaluated at $\left(X_{s}^{t}(x), s\right)$. To estimate the last quantity we will use the definition of $\tau_{x, t}^{\varepsilon}$; if $h \leq \tau_{x, t}^{\varepsilon} \mathcal{P}$ a.s., then $\mathbb{E}\left[\frac{h \wedge \tau_{x, t}^{\varepsilon}-t}{h-t}\right]=1$. Consider the function $\tilde{u}(x, s)=u(x, s)-u_{T}(x)$, and note that this function has the same properties as $u$. Moreover,

$$
\begin{aligned}
\mathcal{P}\left[\tau_{x, t}^{\varepsilon} \leq h\right] & \leq \mathcal{P}\left[\sup _{t \leq s \leq h}\left|\tilde{u}\left(X_{s}^{t}(x), s\right)-\tilde{u}(x, t)\right| \geq \varepsilon\right] \\
& \leq \frac{1}{\varepsilon^{2}} \mathbb{E}\left[\sup _{t \leq s \leq h}\left|\tilde{u}\left(X_{s}^{t}(x), s\right)-\tilde{u}(x, t)\right|\right]^{2} \\
& \leq \frac{\mathrm{C}_{R}}{\varepsilon^{2}} \mathbb{E}\left[(h-t)^{\frac{1}{2}}+\sup _{t \leq s \leq h}\left|X_{s}^{t}(x)-x\right|\right]^{2} \leq \mathrm{C}_{R}^{\prime}(h-t) .
\end{aligned}
$$

Sending $h$ to $t$ we obtain

$$
0 \leq \partial_{t} \phi(x, t)-H\left(x, t, \phi(x, t), \mathbf{x} D \phi(x, t), \mathbf{x} D^{2} \phi(x, t) \mathbf{x}^{T}, \mathcal{J} \phi(x, t)\right),
$$

which is the desired subsolution inequality.

The uniqueness of the viscosity solution of the obstacle problem (4.5)-(4.6) does not follow immediately from the results in Section 2, as the obstacle problem is not directly included in the class described by (1.1)-(1.2). However, as is well known, a comparison principle for an obstacle problem can be easily established starting off from the corresponding comparison principle for the problem without an obstacle, see, for example, [2, 3, 25].

Theorem 4.6. Suppose that assumptions (B) hold. Then the function u given by (4.4) is the unique viscosity solution of the problem (4.5)-(4.6).

Proof. The proof is an extension of Theorem 2.10 to the obstacle problem case.

Let us define $\tilde{u}$ and $\tilde{v}$ as in (2.2)-(2.3) respectively. We would like to prove the comparison result

$$
\tilde{u} \leq \tilde{v} \text { on } \tilde{\Pi} \times[0, T],
$$

arguing by contradiction. Let us suppose that

$$
\mathcal{M}=\sup _{(x, t) \in \tilde{\Pi} \times[0, T]}\{\tilde{u}(x, t)-\tilde{v}(x, t)\}>0 .
$$

An a priori bound on $\mathcal{M}$ is not given, therefore we proceed with the approximated function

$$
\tilde{u}_{\delta}(x, t)=\tilde{u}(x, t)-\delta h_{o}(x), \quad \tilde{v}_{\delta}(x, t)=\tilde{v}(x, t)+\delta h_{o}(x) .
$$

Defining the function

$$
\Psi_{\delta}(x, t)=\tilde{u}_{\delta}(x, t)-\tilde{v}_{\delta}(x, t), \quad(x, t) \in \tilde{\Pi} \times[0, T],
$$

it can be proved that there exists an infinitesimal sequence of parameters $\delta=\delta(n)$ such that $\left(x_{\delta}, t_{\delta}\right)$ is a maximum point for $\Psi_{\delta}$, and, as $\delta \rightarrow 0$,

$$
\tilde{u}_{\delta}\left(x_{\delta}, t_{\delta}\right)-\tilde{v}_{\delta}\left(x_{\delta}, t_{\delta}\right) \rightarrow \mathcal{M}, \quad \delta h_{o}\left(x_{\delta}\right) \rightarrow 0 .
$$

Using Definition 4.1, Lemma 2.12 holds even in the case of the obstacle problem. To get the desired contradiction, we double the $x$ variable and consider the function

$$
(x, y, t) \mapsto \tilde{u}_{\delta}(x, t)-\tilde{v}_{\delta}(y, t)-\psi(x, y),
$$


where $\psi(x, y)$ is defined in (2.11). Using classical arguments in the theory of viscosity solutions, for any fixed $\delta, \varepsilon>0$, this function attains its maximum at some point $\left(x_{\delta}^{\varepsilon}, y_{\delta}^{\varepsilon}, t_{\delta}^{\varepsilon}\right) \in$ $\tilde{\Pi}^{2} \times[0, T]$ such that

$$
\begin{gathered}
x_{\delta}^{\varepsilon}, y_{\delta}^{\varepsilon} \rightarrow x_{\delta}, \quad t_{\delta}^{\varepsilon} \rightarrow t_{\delta}, \quad \frac{1}{2 \varepsilon}\left|x_{\delta}^{\varepsilon}-y_{\delta}^{\varepsilon}\right|^{2} \rightarrow 0, \\
\tilde{u}_{\delta}\left(x_{\delta}^{\varepsilon}, t_{\delta}^{\varepsilon}\right)-\tilde{v}_{\delta}\left(y_{\delta}^{\varepsilon}, t_{\delta}^{\varepsilon}\right) \rightarrow \tilde{u}_{\delta}\left(x_{\delta}, t_{\delta}\right)-\tilde{v}_{\delta}\left(y_{\delta}, t_{\delta}\right),
\end{gathered}
$$

as $\varepsilon \rightarrow 0$. Hence we can suppose, without loss of generality, that for all $\delta, \varepsilon>0$

$$
\begin{array}{cc}
x_{\delta}^{\varepsilon}, y_{\delta}^{\varepsilon} \in \mathcal{Q}\left(r_{\delta}\right), & t_{\delta}^{\varepsilon}<T, \\
\left|u_{\delta}\left(x_{\delta}^{\varepsilon}, t_{\delta}^{\varepsilon}\right)\right|,\left|v_{\delta}\left(y_{\delta}^{\varepsilon}, t_{\delta}^{\varepsilon}\right)\right| \leq R_{\delta}, & \tilde{u}_{\delta}\left(x_{\delta}^{\varepsilon}, t_{\delta}^{\varepsilon}\right)-\tilde{v}_{\delta}\left(y_{\delta}^{\varepsilon}, t_{\delta}^{\varepsilon}\right)>0,
\end{array}
$$

where $r_{\delta}>1$ and $R_{\delta}>0$ do not depend on $\varepsilon$. To simplify notations in what follows, the indices $\delta, \varepsilon$ are replaced with an over-bar and time dependence is omitted.

By an extension of Lemma $2.12, \tilde{u}_{\delta}, \tilde{v}_{\delta}$ solve

$$
\begin{aligned}
& \min \left(-\partial_{t} \tilde{u}_{\delta}+H\left(x, t, \tilde{u}_{\delta}, \mathbf{x} D \tilde{u}_{\delta}, \mathbf{x} D^{2} \tilde{u}_{\delta} \mathbf{x}^{T}, \mathcal{J} \tilde{u}_{\delta}\right)+\delta h_{o}(x)-2 \omega\left(\mathrm{D} \delta h_{o}(x)\right),\right. \\
& \left.\tilde{u}_{\delta}(x, t)-u_{T}(x)\right)=0 \\
& \min \left(-\partial_{t} \tilde{v}_{\delta}+H\left(x, t, \tilde{v}_{\delta}, \mathbf{x} D \tilde{v}_{\delta}, \mathbf{x} D^{2} \tilde{v}_{\delta} \mathbf{x}^{T}, \mathcal{J} \tilde{v}_{\delta}\right)-\delta h_{o}(x)+2 \omega\left(\mathrm{D} \delta h_{o}(x)\right),\right. \\
& \left.\tilde{v}_{\delta}(x, t)-u_{T}(x)\right)=0
\end{aligned}
$$

Proceeding as in Section 2 there exist $\bar{\tau} \in \mathbb{R}$ and symmetric matrices $\bar{M}$ and $\bar{N}$ such that

$$
\left(\begin{array}{cc}
\bar{M} & 0 \\
0 & -\bar{N}
\end{array}\right) \leq \frac{3}{\varepsilon}\left(\begin{array}{cc}
I & -I \\
-I & I
\end{array}\right)
$$

and

$$
\begin{aligned}
\min \left(-\bar{\tau}+H\left(\bar{x}, \tilde{u}_{\delta}, \overline{\mathbf{x} p}, \overline{\mathbf{x}} \bar{M} \overline{\mathbf{x}}^{T}, \hat{\mathcal{J}}_{\kappa} \psi(\bar{x}, \bar{y})+\hat{\mathcal{J}}^{\kappa}\left(\tilde{u}_{\delta}, \bar{p}\right)(\bar{x})\right)\right. & \\
\left.+\delta h_{o}(\bar{x})-2 \omega\left(\mathrm{D} \delta h_{o}(\bar{x})\right), \tilde{u}_{\delta}(\bar{x})-u_{T}(\bar{x})\right) & \leq 0, \\
\min \left(-\bar{\tau}+H\left(\bar{y}, \tilde{v}(\bar{y}), \overline{y p}, \overline{\mathbf{y}} \bar{N} \overline{\mathbf{y}}^{T},-\hat{\mathcal{J}}_{\kappa} \psi(\bar{x}, \bar{y})+\hat{\mathcal{J}}^{\kappa}\left(\tilde{v}_{\delta}, \bar{p}\right)(\mathbf{y})\right)\right. & \\
\left.-\delta h_{o}(\mathbf{y})+2 \omega\left(\mathrm{D} \delta h_{o}(\underline{y})\right), \tilde{v}_{\delta}(\bar{y})-u_{T}(\bar{y})\right) & \geq 0,
\end{aligned}
$$

where $\bar{p}=\frac{1}{\varepsilon}(\bar{x}-\bar{y})$. Subtracting the two inequalities yields

$$
\min \left(\bar{H}_{\kappa}-\bar{G}, \tilde{u}(\bar{x})-\tilde{v}(\bar{y})\right) \leq 0,
$$

where

$$
\begin{aligned}
\bar{H}_{\kappa}= & H\left(\bar{x}, \tilde{u}_{\delta}(\bar{x}), \overline{\mathbf{x}} p, \overline{\mathbf{x}} \bar{M} \overline{\mathbf{x}}^{T}, \hat{\mathcal{J}}_{\kappa} \psi(\bar{x}, \bar{y})+\hat{\mathcal{J}}^{\kappa}\left(\tilde{u}_{\delta}, \bar{p}\right)(\bar{x})\right) \\
& -H\left(\bar{y}, \tilde{v}(\bar{y}), \overline{\mathbf{y}} \bar{p}, \overline{\mathbf{y}} \bar{N} \overline{\mathbf{y}}^{T},-\hat{\mathcal{J}}_{\kappa} \psi(\bar{x}, \bar{y})+\hat{\mathcal{J}}^{\kappa}\left(\tilde{v}_{\delta}, \bar{p}\right)(\bar{y})\right), \\
\bar{G}= & -\delta\left[h_{o}(\bar{x})+h_{o}(\bar{y})\right]+2 \omega\left(\mathrm{D} \delta h_{o}(\bar{x})\right)+2 \omega\left(\mathrm{D} \delta h_{o}(\bar{y})\right) .
\end{aligned}
$$

From now on the proof proceeds as in Section 2, and the desired contradiction $\mathcal{M} \leq 0$ is obtained.

\section{ACKnowledgements}

K. H. Karlsen is supported in part by the BeMatA program of the Research Council of Norway and the European network HYKE, funded by the EC as contract HPRN-CT-200200282. We are grateful to an anonymous referee for very careful reading of the first version of this paper. 


\section{REFERENCES}

[1] O. Alvarez and A. Tourin Viscosity solutions of nonlinear integro-differential equations. Ann. Inst. H. Poincaré Anal. Non Linéaire, 13(3):293-317, 1996.

[2] A. L. Amadori. The obstacle problem for nonlinear integro-differential operators arising in option pricing. Quaderno IAC Q21-000, 2000.

[3] A. L. Amadori. Nonlinear integro-differential evolution problems arising in option pricing: a viscosity solutions approach. Journal of Differential and Integral Equations, 16(7):787-811, 2003

[4] G. Barles, R. Buckdahn, and E. Pardoux. Backward stochastic differential equations and integral-partial differential equations. Stochastics Stochastics Rep., 60(1-2):57-83, 1997.

[5] G. Barles and E. Rouy. A strong comparison result for the Bellman equation arising in stochastic exit time control problems and its applications. Commun. Partial Differ. Equations, 23(11-12):1995-2033, 1998.

[6] F. E. Benth, K. H. Karlsen, and K. Reikvam. Optimal portfolio management rules in a non-Gaussian market with durability and intertemporal substitution. Finance Stoch., 5(4):447-467, 2001.

[7] F. E. Benth, K. H. Karlsen, and K. Reikvam. Optimal portfolio selection with consumption and nonlinear integro-differential equations with gradient constraint: a viscosity solution approach. Finance Stoch., 5(3):275-303, 2001.

[8] F. E. Benth, K. H. Karlsen, and K. Reikvam. Portfolio optimization in a Lévy market with intertemporal substitution and transaction costs. Stoch. Stoch. Rep., 74(3-4):517-569, 2002.

[9] J. Bertoin. Lévy processes, volume 121 of Cambridge Tracts in Mathematics. Cambridge University Press, Cambridge, 1996.

[10] T. Chan. Pricing contingent claims on stocks driven by Lévy processes. Ann. Appl. Probab., 9(2):504$528,1999$.

[11] M. G. Crandall, H. Ishii, and P. Lions. Users' guide to viscosity solutions of second order partial differential equations. Bull. Amer. Math. Soc., 27:1-67, 1992.

[12] W. H. Fleming and H. M. Soner. Controlled Markov processes and viscosity solutions. Springer-Verlag, New York, 1993.

[13] T. Fujiwara and H. Kunita. Stochastic differential equations of jump type and Lévy processes in diffeomorphisms group. J. Math. Kyoto Univ., 25(1):71-106, 1985.

[14] M. G. Garroni and J.-L. Menaldi. Green functions for second order parabolic integro-differential problems, volume 275 of Pitman Research Notes in Mathematics Series. Longman Scientific \& Technical, Harlow, 1992.

[15] M. G. Garroni and J. L. Menaldi. Second order elliptic integro-differential problems, volume 430 of Chapman \& Hall/CRC Research Notes in Mathematics. Chapman \& Hall/CRC, Boca Raton, FL, 2002.

[16] E. R. Jakobsen and K. H. Karlsen. A "maximum principle for semicontinuous functions" applicable to integro-partial differential equations. Preprint, 2003.

[17] I. Karatzas and S. E. Shreve. Brownian motion and stochastic calculus. Springer-Verlag, New York, 1991.

[18] N. Ikeda and S. Watanabe. Stochastic differential equations and diffusion processes, volume 24 of North-Holland Mathematical Library. North-Holland Publishing Co., Amsterdam, 1981.

[19] D. Lamberton and B. Lapeyre. Introduction au calcul stochastique appliqué à la finance. Ellipses Édition Marketing, Paris, 1997.

[20] J. Ma and J. Yong. Forward-backward stochastic differential equations and their applications, volume 1702 of Lecture Notes in Mathematics. Springer, 1999.

[21] R. Mikulyavichyus and G. Pragarauskas. Nonlinear potentials of the Cauchy-Dirichlet problem for the Bellman integro-differential equation. Liet. Mat. Rink., 36(2):178-218, 1996.

[22] M. K. V. Murthy and G. Stampacchia. Boundary value problems for some degenerate-elliptic operators. Ann. Mat. Pura Appl. (4), 80: 1-122, 1968.

[23] É. Pardoux and S. Peng. Backward stochastic differential equations and quasilinear parabolic partial differential equations. In Stochastic partial differential equations and their applications (Charlotte, NC, 1991), pages 200-217. Springer, Berlin, 1992.

[24] É. Pardoux and S. G. Peng. Adapted solution of a backward stochastic differential equation. Systems Control Lett., 14(1):55-61, 1990.

[25] H. Pham. Optimal stopping of controlled jump diffusion processes: a viscosity solution approach. $J$. Math. Systems Estim. Control, 8(1):27 pp. (electronic), 1998.

[26] S. Raible. Lèvy Processes in Finance: Theory, Numerics, and Empirical Facts. PhD thesis, Mathematischen Fakultät der Albert-Ludwigs-Universität Freiburg i. Br., 2000.

[27] A. Sayah. Équations d'Hamilton-Jacobi du premier ordre avec termes intégro-différentiels. I. Unicité des solutions de viscosité. Comm. Partial Differential Equations, 16(6-7):1057-1074, 1991.

[28] A. Sayah. Équations d'Hamilton-Jacobi du premier ordre avec termes intégro-différentiels. II. Existence de solutions de viscosité. Comm. Partial Differential Equations, 16(6-7):1075-1093, 1991. 
[29] M. Schweizer. On the minimal martingale measure and the Föllmer-Schweizer decomposition. Stochastic Anal. Appl., 13(5):573-599, 1995.

[30] H. M. Soner. Optimal control with state-space constraint. II. SIAM J. Control Optim., 24(6):1110-1122, 1986.

[31] H. M. Soner. Optimal control of jump-Markov processes and viscosity solutions. Stochastic differential systems, stochastic control theory and applications (Minneapolis, Minn., 1986), IMA Vol. Math. Appl., 10, 501-511, Springer, New York, 1988.

[32] S. J. Tang and X. J. Li. Necessary conditions for optimal control of stochastic systems with random jumps. SIAM J. Control Optim., 32(5):1447-1475, 1994.

[33] M. A. Vivaldi. Existence and uniqueness results for degenerate-elliptic integro-differential problems, in Elliptic and parabolic problems (Pont-à-Mousson, 1994), 213-223, Volume 325 in Pitman Res. Notes Math. Ser., Longman Sci. Tech., Harlow, 1995,

(Anna Lisa Amadori)

istituto per le Applicazioni del Calcolo "M. Picone"

Consiglio Nazionale Delle Ricerche

Via dEL POLICLINICO, 137

I-00161 Roma, ItALY

E-mail address: a.amadori@iac.cnr.it

$U R L:$ http://www.iac.cnr.it/ amadori/

(Kenneth H. Karlsen)

Department of Mathematics

UNIVERSITY OF BERGEN

JOHS. BRUNSGT. 12

N-5008 BERGEN, NORWAY

AND

Centre of Mathematics for Applications (CMA)

Department OF MATHEMATiCS

UNIVERSITY OF OSLO

P.O. BOX 1053, BLINDERN

N-0316 OSLO, NoRWAY

E-mail address: kennethk@mi.uib.no

$U R L:$ http://www.mi.uib.no/ "kennethk/

(Claudia La Chioma)

Universitá di ROMA "LA SAPIENZA"

Dipartimento di Matematica Pura ed Applicata

Piazzale Aldo Moro, 2

I-00161 Roma, ItALy

E-mail address: c.lachioma@iac.cnr.it

$U R L:$ http://www.iac.cnr.it/ lachioma/ 